\title{
Generic acquisition protocol for quantitative MRI of the spinal cord
}

J. Cohen-Adad ${ }^{1,2}$, E. Alonso-Ortiz ${ }^{3}$, M. Abramovic ${ }^{4}$, C. Arneitz ${ }^{5}$, N. Atcheson ${ }^{6}$, L. Barlow ${ }^{7}$, Robert L. Barry ${ }^{8,9,10}$, M. Barth ${ }^{11}$, M. Battiston ${ }^{12}$, C. Büchel ${ }^{13}$, M. Budde ${ }^{14}$, V.Callot ${ }^{15,16}$, A. Combes ${ }^{17}$, B. De Leener $^{18,19,20}$, M Descoteaux ${ }^{21,22}$, P. L. De Sousa ${ }^{23}$, M. Dostal ${ }^{24}$, J. Doyon ${ }^{25}$, A. Dvorak ${ }^{26}$, F. Eippert $^{27}$, Karla R. Epperson ${ }^{28}$, K.S. Epperson ${ }^{29}$, P. Freund ${ }^{30}$ J. Finsterbusch ${ }^{31}$, A. Foias ${ }^{32}$, M.

\footnotetext{
${ }^{1}$ NeuroPoly Lab, Institute of Biomedical Engineering, Polytechnique Montreal, Montreal, QC, Canada

${ }^{2}$ Functional Neuroimaging Unit, CRIUGM, University of Montreal, Montreal, QC, Canada.

${ }^{3}$ NeuroPoly Lab, Institute of Biomedical Engineering, Polytechnique Montreal, Montreal, QC, Canada

${ }^{4}$ Department of Radiology, Swiss Paraplegic Centre, Nottwil, Switzerland

${ }^{5}$ Department of Radiology, Swiss Paraplegic Centre, Nottwil, Switzerland

${ }^{6}$ Centre for Advanced Imaging, The University of Queensland, Brisbane, Australia

${ }^{7}$ Department of Radiology, University of British Columbia, Vancouver, BC, Canada

${ }^{8}$ Athinoula A. Martinos Center for Biomedical Imaging, Department of Radiology, Massachusetts General Hospital, Charlestown, MA, USA

${ }^{9}$ Department of Radiology, Harvard Medical School, Boston, MA, USA

${ }^{10}$ Harvard-Massachusetts Institute of Technology Health Sciences \& Technology, Cambridge, MA, USA

${ }^{11}$ School of Information Technology and Electrical Engineering, The University of Queensland, Brisbane, Australia

${ }^{12}$ Queen Square MS Centre, Queen Square Institute of Neurology, Faculty of Brain Sciences, University College London, London, United Kingdom

13 Department of Systems Neuroscience, University Medical Center Hamburg-Eppendorf, Hamburg, Germany

${ }^{14}$ Department of Neurosurgery, Medical College of Wisconsin, Milwaukee, WI, USA

${ }^{15}$ Aix-Marseille Univ, CNRS, CRMBM, Marseille, France

${ }^{16}$ APHM, Hopital Universitaire Timone, CEMEREM, Marseille, France

${ }^{17}$ Vanderbilt University Institute of Imaging Science, Vanderbilt University Medical Center, Nashville, TN, USA

${ }^{18}$ Department of Computer and Software Engineering, Polytechnique Montreal, Montreal Canada

${ }^{19} \mathrm{CHU}$ Sainte-Justine Research Centre, Montreal, QC, Canada

${ }^{20}$ Montreal Neurological Institute, McGill University, Montreal, QC, Canada

${ }^{21}$ Centre de Recherche CHUS, CIMS, Sherbrooke, Canada

${ }^{22}$ Sherbrooke Connectivity Imaging Lab (SCIL), Computer Science department, Université de Sherbrooke

${ }^{23}$ Université de Strasbourg, CNRS, ICube, FMTS, Strasbourg, France

${ }^{24}$ UHB - University Hospital Brno, Department of Radiology and Nuclear Medicine, Czech Republic

${ }^{25}$ Montreal Neurological Institute, McGill University, Montreal, QC, Canada

${ }^{26}$ Department of Physics and Astronomy, University of British Columbia, Vancouver, BC, Canada

${ }^{27}$ Max Planck Institute for Human Cognitive and Brain Sciences, Leipzig, Germany

${ }^{28}$ Richard M. Lucas Center, Stanford University School of Medicine, Stanford, CA USA

${ }^{29}$ Richard M. Lucas Center, Stanford University School of Medicine, Stanford, CA USA

${ }^{30}$ Spinal Cord Injury Center Balgrist, University of Zurich, Switzerland

31 Department of Systems Neuroscience, University Medical Center Hamburg-Eppendorf, Hamburg, Germany

${ }^{32}$ NeuroPoly Lab, Institute of Biomedical Engineering, Polytechnique Montreal, Montreal, QC, Canada
} 
Fratini $^{33,34}$, I. Fukunaga ${ }^{35}$, C. A. M. Gandini Wheeler-Kingshott ${ }^{36,37,38}$, G. Germani ${ }^{39}$, G. Gilbert ${ }^{40}$, F. Giove ${ }^{41,42}$, C. Gros ${ }^{43,44}$, F. Grussu ${ }^{45,46}$, A. Hagiwara ${ }^{47}$, P-G. Henry ${ }^{48}$, T. Horák ${ }^{49}$, M. Hori ${ }^{50}$, J. Joers $^{51}$, K. Kamiya ${ }^{52}$, H. Karbasforoushan ${ }^{53}$, M. Kerkovsky ${ }^{54}$, A. Khatibiji5, ${ }^{55,}$ J.-W. Kim ${ }^{57}$, N. Kinany $^{58}$, H.H. Kitzler ${ }^{59}$, S. Kolind ${ }^{60}$, Y. Kong ${ }^{61,62,63}$, P. Kudlička ${ }^{64}$, P. Kuntke ${ }^{65}$, N.D. Kurniawann ${ }^{66}$,

${ }^{33}$ CNR-Nanotec, Rome, Italy

${ }^{34}$ IRCCS Santa Lucia Foundation, Rome, Italy

${ }^{35}$ Department of Radiology, Juntendo University School of Medicine, Tokyo, Japan

${ }^{36}$ Queen Square MS Centre, Queen Square Institute of Neurology, Faculty of Brain Sciences, University College London, London, United Kingdom

${ }^{37}$ Department of Brain and Behavioural Sciences, University of Pavia, Pavia, Italy

${ }^{38}$ Brain MRI 3T Research Centre, IRCCS Mondino Foundation, Pavia, Italy

${ }^{39}$ Neuroradiology Unit, IRCCS Mondino Foundation, Pavia, Italy

${ }^{40}$ MR Clinical Science, Philips Healthcare, Markham, ON, Canada

${ }^{41}$ CREF - Museo storico della fisica e Centro studi e ricerche Enrico Fermi, Rome, Italy

${ }^{42}$ IRCCS Santa Lucia, Rome, Italy

${ }^{43}$ NeuroPoly Lab, Institute of Biomedical Engineering, Polytechnique Montreal, Montreal, QC, Canada

${ }^{44}$ Centre for Advanced Imaging, The University of Queensland, Brisbane, Australia

${ }^{45}$ Queen Square MS Centre, Queen Square Institute of Neurology, Faculty of Brain Sciences, University College London, London, United Kingdom

${ }^{46}$ Radiomics Group, Vall d'Hebron Institute of Oncology, Vall d'Hebron Barcelona Hospital Campus, Barcelona (Spain)

${ }^{47}$ Department of Radiology, Juntendo University School of Medicine, Tokyo, Japan

${ }^{48}$ Center for Magnetic Resonance Research, Department of Radiology, University of Minnesota, Minneapolis, MN, USA

${ }^{49}$ CEITEC - Central European Institute of Technology, Brno, Czech Republic

${ }^{50}$ Department of Radiology, Juntendo University School of Medicine, Tokyo, Japan

${ }^{51}$ Center for Magnetic Resonance Research, Department of Radiology, University of Minnesota, Minneapolis, MN, USA

${ }^{52}$ Department of Radiology, the University of Tokyo, Tokyo, Japan

${ }^{53}$ Interdepartmental Neuroscience Program, Northwestern University School of Medicine, Chicago, IL, USA

${ }^{54}$ UHB - University Hospital Brno, Department of Radiology and Nuclear Medicine, Czech Republic

${ }^{55}$ Department of Neurology and Neurosurgery, McGill University, Montreal, Canada

${ }^{56}$ Centre of Precision Rehabilitation for Spinal Pain (CPR Spine), School of Sport, Exercise and Rehabilitation Sciences, College of Life and Environmental Sciences, University of Birmingham, Edgbaston, Birmingham, United Kingdom

${ }^{57}$ BioMedical Engineering and Imaging Institute (BMEII), Department of Radiology, Icahn School of Medicine at Mount Sinai, New York, NY, USA

${ }^{58}$ Center for Neuroprosthetics, Institute of Bioengineering, Ecole Polytechnique Fédérale de Lausanne, Lausanne, Switzerland

${ }^{59}$ Institute of Diagnostic and Interventional Neuroradiology, Carl Gustav Carus University Hospital, Technische Universität Dresden, Dresden, Germany

${ }^{60}$ Depts. Of Medicine (Neurology), Physics \& Astronomy, Radiology; University of British Columbia, Vancouver, BC, Canada

${ }^{61}$ CAS Key Laboratory of Behavioral Science, Institute of Psychology, Chinese Academy of Sciences, Beijing, China

${ }^{62}$ Department of Psychology, University of Chinese Academy of Sciences, Beijing, China

${ }^{63}$ Wellcome Centre for Integrative Neuroimaging, University of Oxford, UK

${ }^{64}$ CEITEC - Central European Institute of Technology, Brno, Czech Republic

${ }^{65}$ Institute of Diagnostic and Interventional Neuroradiology, Carl Gustav Carus University Hospital, Technische Universität Dresden, Dresden, Germany

${ }^{66}$ Centre for Advanced Imaging, The University of Queensland, Brisbane, Australia 
S. Kusmia ${ }^{67,68,69}$, R. Labounek ${ }^{70,71}$, M.M. Laganà ${ }^{72}$, C. Laule ${ }^{73}$, Christine S. Law ${ }^{74}$, C. Lenglet ${ }^{75}$, T. Leutritz $^{76}$, Y. Liu ${ }^{77,78}$, S. Llufriu ${ }^{79}$, Sean Mackey ${ }^{80}$, E. Martinez-Heras ${ }^{81}$, L. Mattera ${ }^{82}$, I. Nestrasil ${ }^{83,}$ ${ }^{84}$, Kristin P. O'Grady ${ }^{85,86}$, N. Papinutto ${ }^{87}$, D. Papp ${ }^{88}$, D. Pareto ${ }^{89}$, T.B. Parrish ${ }^{90}$, A. Pichiecchio ${ }^{91,92}$

${ }^{67}$ CUBRIC, Cardiff University, Wales, UK

${ }^{68}$ Centre for Medical Image Computing (CMIC), University College London, London, United Kingdom

${ }^{69}$ Epilepsy Society MRI Unit, Chalfont St Peter, United Kingdom

70 Division of Clinical Behavioral Neuroscience, Department of Pediatrics, University of Minnesota, Minneapolis, MN, USA

${ }^{71}$ Departments of Neurology and Biomedical Engineering, University Hospital Olomouc, Olomouc, Czech Republic

${ }^{72}$ IRCCS Fondazione Don Carlo Gnocchi ONLUS, Milan, Italy

${ }^{73}$ Departments of Radiology, Pathology \& Laboratory Medicine, Physics \& Astronomy; International Collaboration on Repair Discoveries (ICORD), University of British Columbia, Vancouver, BC, Canada

${ }^{74}$ Systems Neuroscience and Pain Lab, Department of Anesthesiology, Perioperative and Pain Medicine, Stanford University School of Medicine, Stanford, CA, USA

75 Center for Magnetic Resonance Research, Department of Radiology, University of Minnesota, Minneapolis, MN, USA

${ }^{76}$ Max Planck Institute for Human Cognitive and Brain Sciences, Leipzig, Germany

${ }^{77}$ Department of Radiology, Beijing Tiantan Hospital, Capital Medical University, Beijing, China

${ }_{78}$ Tiantan Image Research Center, China National Clinical Research Center for Neurological Diseases, Beijing, China

${ }^{79}$ Center of Neuroimmunology, Laboratory of Advanced Imaging in Neuroimmunological Diseases, Hospital Clinic Barcelona, Institut d'Investigacions Biomèdiques August Pi i Sunyer (IDIBAPS) and Universitat de Barcelona, Barcelona, Spain.

${ }^{80}$ Systems Neuroscience and Pain Lab, Department of Anesthesiology, Perioperative and Pain Medicine, Stanford University School of Medicine, Stanford, CA, USA

81 Center of Neuroimmunology, Laboratory of Advanced Imaging in Neuroimmunological Diseases, Hospital Clinic Barcelona, Institut d'Investigacions Biomèdiques August Pi i Sunyer (IDIBAPS) and Universitat de Barcelona, Barcelona, Spain.

${ }^{82}$ Fondation Campus Biotech Genève, 1202, Geneva, Switzerland

83 Center for Magnetic Resonance Research, Department of Radiology, University of Minnesota, Minneapolis, MN, USA

84 Division of Clinical Behavioral Neuroscience, Department of Pediatrics, University of Minnesota, Minneapolis, MN, USA

${ }^{85}$ Department of Radiology, Vanderbilt University Medical Center, Nashville, TN USA

${ }^{86}$ Vanderbilt University Institute of Imaging Science, Vanderbilt University Medical Center, Nashville, TN USA

${ }^{87}$ Department of Neurology, University of California San Francisco, San Francisco, CA, USA

${ }^{88}$ Wellcome Centre For Integrative Neuroimaging, FMRIB, NDCN, University of Oxford, Oxford, United Kingdom

${ }^{89}$ Neuroradiology Section, Vall d'Hebron University Hospital. Barcelona, Spain

${ }^{90}$ Feinberg School of Medicine, Northwestern University, Chicago, IL, USA

${ }^{91}$ Department of Brain and Behavioural Neuroscience, University of Pavia, Pavia, Italy

${ }^{92}$ Neuroradiology Unit, IRCCS Mondino Foundation, Pavia, Italy 
, F. Prados ${ }^{93},{ }^{94}, 95$, À. Rovira ${ }^{96}$, M.J. Ruitenberg ${ }^{97}$, Rebecca S. Samson ${ }^{98}$, G. Savini ${ }^{99}$, M. Seif ${ }^{100}$, Alan C. Seifert ${ }^{101}$, Alex K. Smith ${ }^{102}$, Seth A. Smith ${ }^{103,104}$, Z.A. Smith ${ }^{105}$, E. Solana ${ }^{106}$, Y. Suzuki ${ }^{107}$, G. Tackley $^{108}$, A. Tinnermann ${ }^{109}$, J. Valošek ${ }^{110}$, D. Van De Ville ${ }^{111,112}$, Marios C. Yiannakas ${ }^{113}$, Kenneth A. Weber $I^{114}$, N. Weiskopf ${ }^{115,116}$, R.G. Wise ${ }^{117,118}$, P. O. Wyss ${ }^{119}$, J. $\mathrm{Xu}^{120}$

${ }^{93}$ Queen Square MS Centre, Queen Square Institute of Neurology, Faculty of Brain Sciences, University College London, London, United Kingdom

94 Centre for Medical Image Computing, Medical Physics and Biomedical Engineering Department, University College London, London, United Kingdom

${ }^{95}$ E-health Centre, Universitat Oberta de Catalunya, Barcelona, Spain

${ }^{96}$ Neuroradiology Section, Vall Hebron University Hospital. Barcelona, Spain

${ }^{97}$ School of Biomedical Sciences, Faculty of Medicine, The University of Queensland, Brisbane, Australia

${ }^{98}$ Queen Square MS Centre, Queen Square Institute of Neurology, Faculty of Brain Sciences, University College London, London, United Kingdom

${ }^{99}$ Neuroradiology Unit, IRCCS Mondino Foundation, Pavia, Italy

${ }^{100}$ Spinal Cord Injury Center Balgrist, University of Zurich, Switzerland

101 BioMedical Engineering and Imaging Institute, Department of Radiology, Icahn School of Medicine at Mount Sinai, New York, NY, USA

102 Wellcome Centre For Integrative Neuroimaging, FMRIB, NDCN, University of Oxford, Oxford, United Kingdom

${ }^{103}$ Department of Radiology, Vanderbilt University Medical Center, Nashville, TN USA

${ }^{104}$ Vanderbilt University Institute of Imaging Science, Vanderbilt University Medical Center, Nashville, TN USA

${ }^{105}$ University of Oklahoma Health Sciences Center, Oklahoma City, OK, USA

106 Center of Neuroimmunology, Laboratory of Advanced Imaging in Neuroimmunological Diseases, Hospital Clinic Barcelona, Institut d'Investigacions Biomèdiques August Pi i Sunyer (IDIBAPS) and Universitat de Barcelona, Barcelona, Spain.

${ }^{107}$ Department of Radiology, the University of Tokyo, Tokyo, Japan

${ }^{108}$ CUBRIC, Cardiff University, Wales, UK

109 Department of Systems Neuroscience, University Medical Center Hamburg-Eppendorf, Hamburg, Germany

110 Department of Neurology, Faculty of Medicine and Dentistry, Palacký University and University Hospital Olomouc, Olomouc, Czech Republic

${ }^{111}$ Center for Neuroprosthetics, Institute of Bioengineering, Ecole Polytechnique Fédérale de Lausanne, Lausanne, Switzerland

${ }^{112}$ Department of Radiology and Medical Informatics, University of Geneva, Geneva, Switzerland

${ }^{113}$ Queen Square MS Centre, Queen Square Institute of Neurology, Faculty of Brain Sciences, University College London, London, United Kingdom

114 Systems Neuroscience and Pain Lab, Department of Anesthesiology, Perioperative and Pain Medicine, Stanford University School of Medicine, Stanford, CA, USA

${ }^{115}$ Max Planck Institute for Human Cognitive and Brain Sciences, Leipzig, Germany

${ }^{116}$ Felix Bloch Institute for Solid State Physics, Faculty of Physics and Earth Sciences, Leipzig University, Linnéstraße 5, 04103 Leipzig, Germany

${ }^{117}$ CUBRIC, Cardiff University, Wales, UK

118 Institute for Advanced Biomedical Technologies, Department of Neuroscience, Imaging and Clinical Sciences, "G. D'Annunzio University" of Chieti-Pescara, Italy

${ }^{119}$ Department of Radiology, Swiss Paraplegic Centre, Nottwil, Switzerland

${ }_{120}$ BioMedical Engineering and Imaging Institute, Departments of Radiology and Neuroscience, Icahn School of Medicine at Mount Sinai, New York, NY, USA 


\section{Abbreviations}

CSA - cross-sectional area

CSF - cerebrospinal fluid

DTI - diffusion tensor imaging

DWI - diffusion weighted imaging

FA - fractional anisotropy

GM - gray matter

FOV - field of view

MD - mean diffusivity

MT - magnetization transfer

MTR - magnetization transfer ratio

qMRI - quantitative magnetic resonance imaging

SAR - specific absorption rate

SC - spinal cord

SCT - spinal cord toolbox

SNR - signal-to-noise ratio

WM - white matter 


\section{Abstract}

Quantitative spinal cord (SC) magnetic resonance imaging (MRI) is fraught with challenges, among which is the lack of standardized imaging protocols. Here we present a prospectively harmonized quantitative MRI protocol, which we refer to as the spine generic protocol, for the three main 3T MRI vendors: GE, Philips and Siemens. The protocol provides valuable metrics for assessing SC macrostructural and microstructural integrity: T1-weighted and T2-weighted imaging for SC cross-sectional area (CSA) computation, multi-echo gradient echo for gray matter CSA, as well as magnetization transfer and diffusion weighted imaging for assessing white matter microstructure. The spine generic protocol was used to acquire data across 42 centers in 260 healthy subjects, as detailed in the companion paper [REF-DATA]. The spine generic protocol is open-access and its latest version can be found at: https://spinalcordmri.org/protocols. The protocol will serve as a valuable starting point for researchers and clinicians implementing new SC imaging initiatives.

Note to the reviewer/editor/publisher: the companion paper is referred to as [REF-DATA] 


\section{Introduction}

Quantitative MRI (qMRI) aims to provide objective continuous metrics that specifically reflect the morphology, microstructure and/or chemical composition of tissues ${ }^{1,2}$, thereby enabling deeper insight and understanding of disease pathophysiology. While qMRI techniques have been successfully implemented in the brain for several decades, they remain largely underutilized for spinal cord (SC) imaging in both clinical and research settings, mostly as a direct consequence of the many challenges that need to be overcome in order to acquire good quality data ${ }^{3,4}$.

For the past 20 years, researchers have been developing methods to overcome the challenges around SC imaging, including more sensitive coil arrays ${ }^{5,6}$ and advanced pulse sequences for mitigating motion and susceptibility artifacts ${ }^{4,7}$. As a result, it is now possible to acquire state-of-the-art SC qMRI data that have a strong potential for providing highly relevant insights into SC anatomy and function. However, a remaining issue is that there is no clear consensus within the imaging community for acquiring SC qMRI data, leading to (i) wasted time and money spent on pilot scans for every new SC research initiative, and (ii) a large variability in imaging parameters for multi-site, multi-vendor studies, hampering statistics for assessing biomarkers.

\section{Development of the Protocol}

The present study gathered a consortium of international SC researchers to provide a prospectively harmonized consensus protocol for acquiring high-quality qMRI of the human cervical SC at 3 Tesla (T) across the three main MRI vendors (GE, Philips and Siemens). We call this the spine generic protocol. QMRI techniques covered in the spine generic protocol (and illustrated in Figure 1) include:

SC cross-sectional area (CSA): The CSA of the whole SC has been shown to be a sensitive biomarker in multiple sclerosis (MS) ${ }^{8-11}$, amyotrophic lateral sclerosis (ALS) ${ }^{12-16}$, X-linked adrenoleukodystrophy (ALD) with myelopathy ${ }^{17}$, and both traumatic or non-traumatic SC injury 18,19. Additionally, SC segmentation is useful for atlas-based analysis ${ }^{20}$.

CSA of the SC gray matter (GM): GM CSA is relevant for diagnosis ${ }^{21}$ and prognosis in ALS ${ }^{16}$. Additionally, delineating the GM is relevant for quantifying pathologies juxtaposing with the GM (e.g. MS lesions), for fMRI applications and for atlas-based analysis.

Magnetization transfer (MT): The MT technique has been shown to be sensitive to demyelination ${ }^{22}$ and has been applied in various SC diseases, such as adrenomyeloneuropathy $(\mathrm{AMN})^{23}$ and $\mathrm{MS}^{24}$, as well as in SC injury ${ }^{25,26}$.

Diffusion tensor imaging (DTI): DTI is one of the simplest diffusion weighted imaging (DWI) models that can quantify microstructural integrity and has been deemed sensitive to degeneration and demyelination of SC white matter (WM) tracts in a variety of diseases ${ }^{3,26-29}$ and after SC injury ${ }^{19,27}$. In non-traumatic SC injury, DWI appears to be promising and sensitive to presymptomatic microstructural changes ${ }^{30,31}$. 

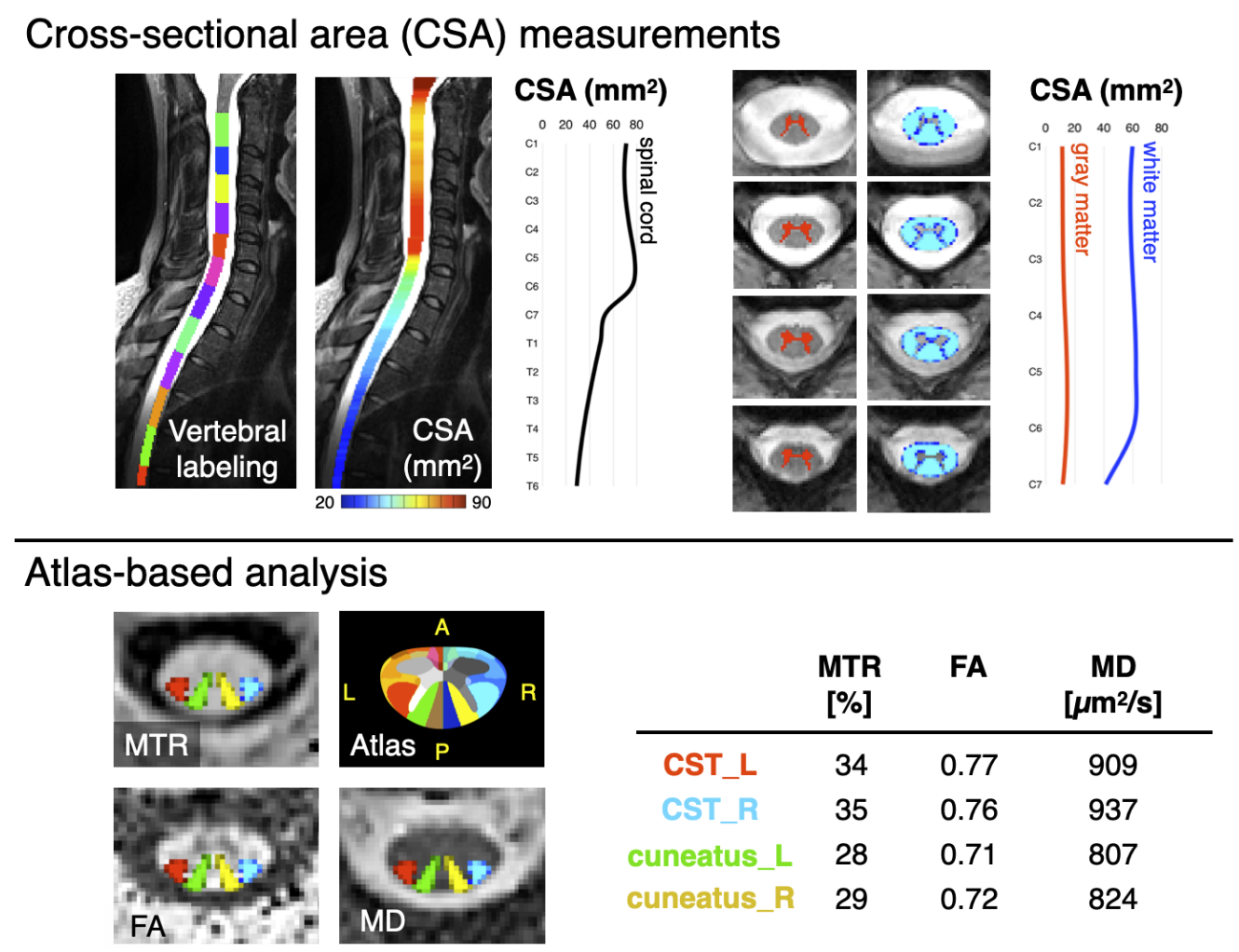

Figure 1. Illustration of the MRI metrics that could be extracted from the spine generic protocol. The top panel "Cross-sectional area (CSA) measurements" shows morphometric measures of the spinal cord and its gray and white matter. The bottom panel "Atlas-based analysis" on the left shows axial views of qMRI maps: Magnetization Transfer Ratio (MTR), Fractional Anisotropy (FA) and Mean Diffusivity (MD), with an overlay of 4 spinal tracts of general interest: the descending corticospinal tract (CST) and the ascending cuneatus, left (L) and right (R). The "Atlas" image corresponds to the white matter atlas ${ }^{32}$, which includes 30 white matter tracts that could be used for computing metrics within specific tracts of interest. This atlas also includes 6 parcellations of the gray matter. The table shows average values of each metric in the corresponding tract.

To demonstrate the practical implementation and reproducibility of the proposed protocol, single-subject and multi-subject datasets were acquired across multiple centers. Relevant qMRI metrics were calculated using a fully-automatic analysis pipeline, and those metrics were compared within site, across sites (within vendor), and across different vendors. Details of the datasets, processing pipelines and generated normative values are available in a joint publication [REF-DATA]. 
When optimizing protocols across vendors, a recurrent question is: Should we minimize the differences in acquisition parameters across vendors, or should we optimize image quality on each platform? The spine generic protocol stands in between: we aimed at minimizing protocol differences in order to facilitate the interpretation of multi-vendor studies, but at the same time we optimized parameters for each vendor separately when the hardware or software enabled it. For example, on the DWI protocol, the TE was always minimized in order to maximize SNR, which minimally affects the diffusion-specific signal (the b-value was kept the same). Given that platforms are equipped with different gradient nominal strength capabilities (ranging from 40 $\mathrm{mT} / \mathrm{m}$ to $80 \mathrm{mT} / \mathrm{m}$ for current clinical systems), this yielded very different TEs depending on the platform. Similarly, some software versions were limited with respect to the minimum achievable TR on MT sequences; again, here the TR was optimized for each system separately, yielding full MT protocols (GRE-MT1/MT0/T1w) that varied from $5.4 \mathrm{~min}$ to $8.9 \mathrm{~min}$, depending on the platform. However, in this case, MTR and MTsat are impacted by TR. This partly explains the discrepancies observed between GE and the two other vendors (see [REF-DATA]).

Because hardware and pulse sequence environments vary across vendors, it will never be possible to obtain the exact same acquisition configuration across vendors. Even within the same vendors, some variability could exist due to the different specifications for different models and the adjustment and maintenance status of individual scanners (acoustic resonances, helium levels, eddy-currents, software patches, etc.). From a practical standpoint, as in the case for the T1w vs. T2w SC CSA (see Figure 11 in [REF-DATA]), the relationship between qMRI metrics obtained from different vendors/models/sites can be modeled as fixed or random effects ${ }^{33}$.

The spine generic protocol has been used (fully, in part or a modified version of it) in the following applications: imaging methods ${ }^{34}$, method development in healthy subjects ${ }^{20,35-48}$, functional MRI ${ }^{49,50}$, MS ${ }^{51-53}$, mucopolysaccharidoses ${ }^{54}$, adrenoleukodystrophy ${ }^{17}$, ALS ${ }^{16,55,56}$, degenerative cervical myelopathy ${ }^{30,31,57-59}$, and stroke ${ }^{60}$.

The spine generic protocol has also been recommended in recent guidelines ${ }^{28,61-64}$ and adopted by multi-center initiatives such as the INSPIRED ${ }^{121}$ and the CanProCo ${ }^{122}$ studies, respectively dealing with cervical myelopathy and MS populations.

\section{Applications of the Method}

The proposed protocol is not geared towards a specific disease and it is suitable for imaging WM pathology (demyelination and Wallerian degeneration via axon/myelin-sensitive

121

https://www.wingsforlife.com/us/research/imaging-spinal-cord-injury-and-assessing-its-predictive-value-th $\frac{\text { e-inspired-study-2675/ }}{122}$

https://mssociety.ca/about-ms-research/about-our-research-program/research-we-fund/canadian-prospect ive-cohort-study-to-understand-progression-in-ms-canproco 
techniques), GM pathology (ALS, via GM CSA quantification), and traumatic and non-traumatic SC injury (structural scans to assess compression and/or to quantify atrophy above/below lesions or injury). Additional clinical scans (e.g. 2D FLAIR, STIR) that are specific to particular diseases and/or are part of the clinical routine can be added at the discretion of the researcher/clinician. Potential clinical uses of this protocol include improved pathological diagnosis, monitoring of disease progression or recovery, and/or prediction of outcomes. For more details on the possible applications see the section "Development of the Protocol".

\section{Comparison with Other Methods}

In this section we discuss alternative techniques to those included in the proposed protocol. These may include techniques that are still at the research stage but could eventually be added to the protocol.

\section{Advanced Shimming}

The default shim coils that are integrated in the MR system are usually not sufficient for compensating for the high spatial variations of the magnetic field across the SC axis, which has motivated the development of custom high-order shim coils ${ }^{65,66}$.

Another approach to further minimize $B_{0}$ inhomogeneities is dynamic shimming, wherein shim coefficients are set for each slice independently. This approach only works for 2D imaging, but is particularly interesting for axial EPI acquisitions. In the case of SC imaging where axial slices are relatively thick $(3-5 \mathrm{~mm})$, the strongest effect of gradient inhomogeneity is along the $z$ direction (superior-inferior axis). Researchers have thus proposed to only correct for gradients along this axis, naming the approach z-shimming ${ }^{67,68}$. This technique has shown substantial improvement in image quality for gradient-echo EPI, notably by reducing signal dropout, which is typically observed at the vicinity of intervertebral discs. For more details on shimming strategies for SC imaging, see ${ }^{69}$.

Another important effect to consider is the dynamic nature of the $\mathrm{B}_{0}$ field, as it varies throughout respiration. This effect is particularly problematic when imaging close to the lungs, e.g. around vertebral levels C7-T1. $B_{0}$ variation can be about $70 \mathrm{~Hz}$ at $3 \mathrm{~T}^{70}$ and $110 \mathrm{~Hz}$ at $7 \mathrm{~T}^{71}$, causing voxel displacement in EPI readout and ghosting in EPI and non-EPI imaging. To tackle this issue, real-time shimming can be used, which consists in varying the shimming gradients during the subject's breathing, as was demonstrated in the brain at $7 \mathrm{~T}^{72}$ and for the spinal cord at 3T ${ }^{66}$. All these advanced techniques are being actively developed, so there is hope that vendors will rapidly translate some of these innovations into widely-available products.

\section{Navigator echoes}

Navigator echoes can be used to measure drift in the $B_{0}$ field, which can then be used to adjust the excitation frequency and/or the filling of k-space. This technique notably helps reduce 
ghosting and is important to ensure proper reconstruction of readout-segmented EPI sequences 73. Navigators are effective for brain scans where most of the region of interest occupies the acquired volume. However, when applied to the SC area, they can create spurious phase offsets. This is because the SC represents a small portion of the acquired volume, shows a relatively poor shim, and is prone to multiple non-linear motions (e.g. muscles move independently of the cord), leading to complex spatio-temporal phase distributions. One approach that seems promising is that of $2 \mathrm{D}$ or $3 \mathrm{D}$ navigators ${ }^{74}$, whereby only a portion of the volume is selected to estimate phase dynamics. 2D or 3D navigator echoes could potentially be incorporated into the DWI sequence that is part of the spine generic protocol.

\section{B1+ mapping}

$\mathrm{RF}$ transmit field $(\mathrm{B} 1+)$ maps are used in $\mathrm{qMRI}$ to correct for $\mathrm{B} 1+$ inhomogeneity-induced signal intensity variation. This can be particularly useful for obtaining accurate T1 ${ }^{75}$ and MT measurements ${ }^{76}$ and has recently been incorporated within the MTsat protocol ${ }^{77}$. B1+ maps can also be used for shimming the RF field in parallel transmit systems ${ }^{78}$, which is of particular interest at ultra-high field strengths where dielectric artifacts present significant challenges for qMRI. B1+ maps can be obtained using a standard product double angle EPI sequence ${ }^{76}$. Some other commonly used methods include magnetization prepared TurboFLASH ${ }^{79}$, spin-echo/stimulated-echo acquisition ${ }^{80}$, actual flip angle imaging ${ }^{81}$, the DREAM sequence ${ }^{82}$ and the Bloch Siegert method ${ }^{83}$. B1+ mapping is not currently included in the spine generic protocol but the protocol could be extended to do so.

\section{Phase sensitive inversion recovery (PSIR)}

In the last decade, sagittal turbo spin echo phase sensitive inversion recovery (PSIR) protocols have been successfully applied to SC imaging of MS patients and those with radiologically isolated syndrome (RIS) for lesion detection ${ }^{84-86}$. In 2013, an axial 3D gradient echo PSIR protocol was successfully applied for the first time in the SC of MS patients using a Philips scanner, showing very good gray matter/white matter/lesion contrast ${ }^{87,88}$. A clinically feasible gradient echo 2D PSIR protocol (about 2 minutes to acquire a single slice) was developed in 2015 on a Siemens 3T scanner ${ }^{89}$. That 2D PSIR protocol has been assessed between the three major MRI vendors ${ }^{37}$ and used to measure in vivo GM and WM areas at multiple SC levels (cervical and thoracic) in MS patients ${ }^{90,91}$, motor neuron disease patients ${ }^{92}$ and healthy controls ${ }^{93,94}$.

\section{Experimental Design}

The spine generic protocol has been optimized and tested for the cervical SC at 3T. In this section we discuss how it could be adapted for the thoraco-lumbar region, or for other field strengths. 


\section{Thoraco-lumbar cord}

While the present protocol is optimized and validated for the cervical cord, most of the sequences proposed here could be ported to the thoraco-lumbar region with minimal or no adjustments. The amount of modification required mostly depends on the RF receive coil that is available. One notable advantage of the cervical region is the possibility of having coil elements around the neck, which provides better performance for accelerated acquisitions (GRAPPA, SENSE) and higher signal-to-noise ratio (SNR). When going to the lower cord, coil elements are typically arranged in a flat fashion, reducing acceleration and SNR. Hence, sequences already suffering from low SNR might need modifications, e.g., a larger voxel size.

In general, the T1w, T2w and MT sequences could likely be applied to the lower cord without modifications. The DWI protocol might require additional averaging and/or larger in-plane voxels to increase the SNR. Furthermore, using saturation bands for inner field of view (FOV) DWI acquisitions may be much more challenging or even impossible due to specific absorption rate (SAR) and saturation band thickness limits. The ME-GRE sequence is feasible ${ }^{95}$ but might require additional averaging ${ }^{96}$, and/or the use of navigator echoes to compensate for respiratory-related ghosting. Protocol optimization could be aided by the use of advanced spinal cord phantoms made of "tissue-like" materials that mimic respiration-related dynamic changes in the $\mathrm{B}_{0}$ field, such as the one proposed by De Tillieux et al. ${ }^{97}$.

\section{Other field strengths}

While the spine generic protocol was optimized and validated at $3 \mathrm{~T}$, only slight modifications would be required to adapt the protocol to $1.5 \mathrm{~T}$ systems. Depending on what researchers would like to do (CSA measurements, lesion quantification, etc.), the SNR and contrast-to-noise ratio would need to be adjusted by finding the right tradeoff between spatial resolution and acquisition time. Relaxation parameters also change at lower and higher fields. For example, tissue T1 is shorter at 1.5T, which could help reduce TR in T1w sequences. Fortunately, SAR is also lower at $1.5 \mathrm{~T}$, which allows one to reduce the TR in SAR-intensive sequences, such as the MT protocol or the T2w sequence (including the DWI sequence).

At 7T, parameters would likely require greater changes than those needed to adapt to $1.5 \mathrm{~T}$. While SNR is higher at $7 \mathrm{~T}$, allowing one to reduce the voxel size, susceptibility effects are also increased. This is particularly problematic for the EPI-based DWI protocol (increased image distortions) ${ }^{98}$ and the long TE gradient echo sequences used for the T2* protocols ${ }^{99}$. Additionally, SAR is higher at $7 \mathrm{~T}$, which leads to challenges when using SAR-intensive sequences such as the MT protocol, the DWI sequence, or the T2w sequence.

More challenges exist when moving between field strengths, including $B 1+$ effects, dynamic $B_{0}$ changes, changes in T1, T2 and T2*, local vs. body RF transmit coils and different safety profiles. Further investigations are needed to properly adapt the spine-generic protocol to other field strengths. 


\section{Expertise needed to implement the protocol}

The protocol could be implemented by a certified MR technician or by a researcher/clinician familiar with MRI acquisition.

\section{Additional remarks and future directions}

The spine generic acquisition protocol is an important milestone for the SC qMRI community. It provides a starting point for researchers and clinicians implementing new SC imaging initiatives, and we believe that this protocol will improve clinical trials involving SC imaging. We would like to stress that the protocol will evolve with new MR hardware and software releases, as well as with research advances such as protocol optimizations and novel pulse sequence developments. Moreover, we are planning to make the protocol available for other vendors (e.g., Canon). For this reason, it is important that researchers using and publishing with this protocol always refer to its release number ${ }^{123}$. The SC MRI community has initiated a forum ${ }^{124}$ to encourage discussions about the generic protocol, how to use it, and how we could further improve it.

We would also like to reiterate that the spine generic protocol is not geared towards a specific disease. Researchers are welcome to tune the proposed protocol by modifying parameters and/or adding/removing sequences as needed. A recent example is the development of a standardized brain and spinal cord MRI protocol for patients with MS ${ }^{100}$.

The present study also comes with two publicly-available datasets (single- and multi-subject) [REF-DATA], which are shared according to the 'Findable, Accessible, Interoperable and Reusable' (FAIR) principles ${ }^{101}$. To the best of our knowledge, these are the first "large-scale" multi-center qMRI SC datasets ever acquired and made public. The multi-subject dataset could be used to create normative qMRI values, serving as age-matched healthy control references. More generally, these datasets will be useful for developing new image processing tools dedicated to the SC, and the fact that they are public makes it possible for researchers to compare tools with the same data.

In a time where reproducibility of scientific results is a major concern ${ }^{102}$, we believe a consensus acquisition protocol along with publicly-shared datasets and a transparent analysis pipeline provide a solid foundation for the field of SC qMRI so that, in the future, inclusion of the $\mathrm{SC}$ in neuroimaging protocols will become a no-brainer.

${ }^{123}$ https://github.com/spine-generic/protocols/releases

124 https://forum.spinalcordmri.org/ 


\section{Materials}

\section{Equipment}

\section{MRI Scanner}

A whole body GE, Philips or Siemens 3T MRI scanner is required.

\section{Coils}

Image quality is largely affected by the receive coil. While most $1.5 \mathrm{~T}$ and $3 \mathrm{~T}$ systems use the integrated body coil for radiofrequency transmission to ensure excellent homogeneity, also referred to as the $\mathrm{B} 1+$ profile, reception can be done with various other coils, each having specific performance characteristics in terms of their sensitivity profile, which defines signal-to-noise ratio (SNR), and g-factor, which describes the parallel imaging capability; i.e., how much one can accelerate (in the phase-encode and slice-select directions) ${ }^{5}$. The recommended receive coils, for specific parts of the spine, are listed in Table 6.

\section{Sequences: Overview}

The required sequences are illustrated in Figure 2 and vendor-specific sequence names are listed in Table 1. All the recommended sequences are available as a product, however, old software versions might not have all up-to-date product sequences, and there may be research sequences that are equivalent. When applicable, this information is mentioned within this manuscript. The protocols (pdf + import files) are freely available at: https://github.com/spine-generic/protocols.

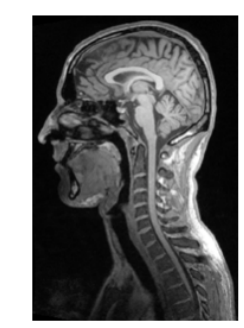

T1w (3D) $1 \mathrm{~mm}$ - Brain \& Spine
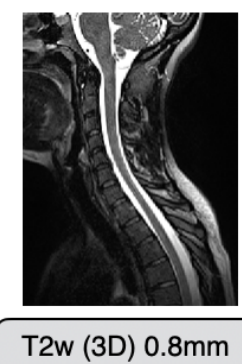
- Cord atrophy

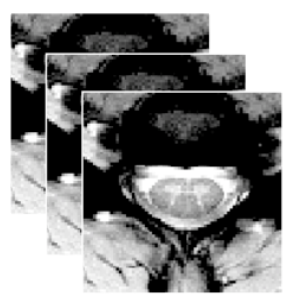

ME-GRE (2D) - Gray matter atrophy

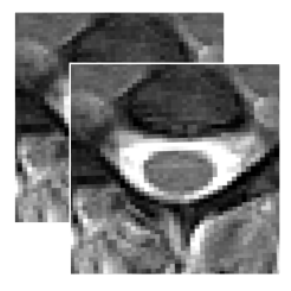

Magnetization Transfer - Demyelination in WM

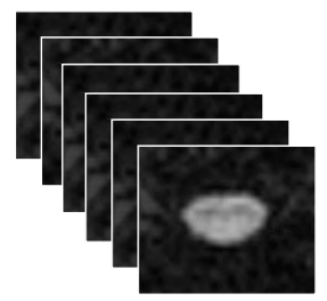

Diffusion Weighted Imaging - Demyelination in WM - Axon degeneration

Figure 2. List of sequences included in the spine generic protocol (in black) with possible applications (in red). The total acquisition time is 20-30min, depending on the vendor/model. 


\begin{tabular}{|c|c|c|c|}
\hline & GE & Philips & Siemens \\
\hline T1w (3D) & BRAVO/IR-FSPGR & T1TFE & MPRAGE \\
\hline $\mathrm{T} 2 \mathrm{w}(3 \mathrm{D})$ & CUBE & VISTA & SPACE \\
\hline ME-GRE ${ }^{125}(2 D)$ & MERGE & mFFE & GRE "medic" \\
\hline GRE-MT1/MT0/T1w (3D) & SPGR & FFE $^{126}$ & GRE \\
\hline DWI (2D) & $\begin{array}{l}\text { SE-EPI with or without } \\
\text { FOCUS }\left({ }^{*}\right)\end{array}$ & Zoom Diffusion( $\left.{ }^{*}\right)$ & $\begin{array}{l}\text { ep2d_diff with or without } \\
\left.\text { ZOOMit( }{ }^{*}\right)\end{array}$ \\
\hline
\end{tabular}

Table 1. List of sequences included in the spine generic protocol. All sequences come by default with the MRI system, except those marked with $\left(^{*}\right)$, which require a special license.

\section{Sequences: General Recommendations}

\section{Shimming}

Shimming refers to homogenizing the static magnetic field $\left(B_{0}\right)$ and is a necessary step for recording reliable images, especially in regions that are prone to large $\mathrm{B}_{0}$ inhomogeneities, such as the SC. Without proper shimming, fat saturation would not work effectively, slice excitation profiles would not be accurate and EPI data could show distortions and signal drop out, with the latter being particularly prevalent in gradient echo imaging, often used in functional MRI studies.

The very first "active" attempt to mitigate susceptibility artifacts is usually performed just before starting an MRI scan via a procedure called active shimming. This procedure consists of estimating a field map and then computing a set of "shim coefficients", i.e. the amount of current that needs to go into each gradient and shim coil in order to minimize the static magnetic field inhomogeneity in a specified "shim adjust volume" (the green box in Figure 8).

\section{Slice orientation}

For 3D acquisitions with isotropic resolution ( $T 1 w$ and $T 2 w$ ), we recommend sagittal acquisition for an efficient S-I coverage with the minimum number of slices required to cover the cord (on the T2w). Note that typical clinical 2D scans use thick sagittal slices, which is popular for diagnosis with T1/T2/STIR/PDw contrasts, but these should not be used for measuring CSA or for template-based analysis due to the poor R-L resolution.

For qMRI methods that produce microstructural metrics (MT, DWI), we recommend axial orientation (orthogonal to the SC) with high in-plane resolution and thick slices. This approach

\footnotetext{
${ }^{125} \mathrm{ME}$ stands for multi-echo

${ }^{126}$ On the Philips system, the MT1 and MT0 scans are acquired within the same sequence, and the MTR is automatically calculated.
} 
takes advantage of the (quasi-) coherently oriented fibers along the S-I direction to increase slice thickness and thus gain SNR. The high in-plane resolution, ideally sub-millimetric, is important for minimizing the partial volume effect between adjacent internal structures (WM tracts, GM), thereby ensuring accurate quantification of metrics. For 2D multislice sequences, if the sequence allows, each individual slice should ideally be orthogonal to the cord ${ }^{103}$. If not possible, slices should be oriented such that the region of most interest is orthogonal to the cord (leaving other regions with larger partial volume effects). Alternatively, if time allows, slices may be separated into several pseudo-contiguous slabs, each orthogonal to the cord and containing 3-5 slices. Note that using thinner slices mitigates the partial volume effect, although this comes at the cost of lower SNR. Thinner slices also mitigate intravoxel dephasing due to inhomogeneities in the static magnetic field, which lead to signal drop-out in gradient-echo imaging ${ }^{104}$. Axial acquisitions with thick slices are also recommended for measuring GM CSA.

\section{Phase-encoding direction}

There are a few considerations to be made when choosing the phase-encoding direction. The advantages of the A-P and R-L directions for a transverse (perpendicular-to-the cord) image orientation can be found in Table 2.

\begin{tabular}{|l|l|}
\hline R-L & \begin{tabular}{l} 
- The cord is less curved along this axis, allowing for a smaller FOV (only if using \\
outer-volume suppression technique) and thus less k-space lines, yielding faster \\
acquisition times in single-line readout schemes and less distortions in EPI. \\
- More robust to poor fat suppression (due to the fat in the posterior neck region). \\
- Less ghosting due to swallowing and pulsatile vessels. \\
\hline - In EPI, susceptibility distortions would not create a R-L asymmetry, which could be \\
problematic in some study designs where the R-L symmetry of the cord is part of the \\
underlying study hypotheses (e.g. comparing diffusion metrics between the left and \\
right CST). \\
- Less prone to peripheral nerve stimulation (although this also depends on the vendor, \\
and how oblique the slices are).
\end{tabular} \\
\hline
\end{tabular}

Table 2. Advantages of $R-L$ and A-P phase-encoding directions.

Reconstruction, interpolation, filters

Interpolation (e.g., via zero filling) is not recommended because it (i) can be done during post-processing, (ii) takes more physical space for MRI raw data, and (iii) can introduce confusion as to what is the native resolution when sharing NIfTI data or publishing results. 


\section{Sequences: Parameters Details}

Justifications for the sequence type and parameters used in the spine generic protocol are summarized below in Table 3, Table 4 and Table 5.

T1w (3D sagittal)

\begin{tabular}{|c|c|}
\hline Purpose & $\begin{array}{l}\text { - Measuring SC CSA and/or volume } \\
\text { - Registering to a template (preferred for disc } \\
\text { labeling) } \\
\text { - Assessing lesions } \\
\text { - Measuring brain atrophy } \\
\text { - Registering to a template registration (preferred for cord } \\
\text { segmentation) } \\
\text { - Assessing lesions and compression }\end{array}$ \\
\hline Pros & $\begin{array}{l}\text { - Efficient SNR per unit time } \\
\text { - High SC/CSF contrast (good for SC segmentation) } \\
\text { - } 320 \mathrm{~mm}^{2} \text { FOV in } \sim 5 \mathrm{~min} \text { at } 1 \mathrm{~mm} \text { iso with full brain } \\
\text { and cervical-spine coverage } \\
\text { - Low specific absorption rate (SAR) } \\
\text { - High WM/GM contrast in the brain (good for } \\
\text { cortical surface segmentation) } \\
\text { - Vertebral discs are well contrasted }\end{array}$ \\
\hline Cons & $\begin{array}{l}\text { - High SAR } \\
\text { - Cannot cover full brain in }<10 \mathrm{~min} \text { at } 0.8 \mathrm{~mm} \text { iso } \\
\text { - Poor visibility of vertebral discs } \\
\text { - More prone to Gibbs ringing artifact at high-contrast SC/CSF } \\
\text { interface }\end{array}$ \\
\hline Parameters & $\begin{array}{l}\text { - Sagittal orientation: rotated about A-P axis for alignment with the cord } \\
\text { - Spatial resolution: } 1 \mathrm{~mm} \text { isotropic (T1w), } 0.8 \mathrm{~mm} \text { isotropic (T2w) } \\
\text { - Slab selective excitation: to avoid R-L aliasing of the body (phase encoding directions on this 3D sequence are R-L } \\
\text { and S-I). } \\
\text { - TR (for T1w): inspired by the Human Connectome Project (HCP) protocol }{ }^{105} \text {, slightly reduced to find a compromise } \\
\text { between satisfactory white/gray matter contrast in the brain and reducing the total acquisition time. } \\
\text { - TR (for T2w): sufficiently high to prevent T1 recovery effects causing signal dropout in the CSF (See Figure } 3 \text { b in } \\
\text { [REF-DATA]), while keeping it low to reduce total acquisition time. } \\
\text { - TE, TI, Flip Angle (for T1w): inspired by the HCP protocol } \\
\text { - TE, Flip Angle (for T2w): inspired by previous studies }{ }^{25,61} \text {, optimized for satisfactory SC/CSF contrast and SAR. }\end{array}$ \\
\hline QC & $\begin{array}{l}\text { - Motion artifact: swallowing, pulsatility, motion (blurriness in A-P direction) } \\
\text { - Homogeneity of signal within the cord } \\
\text { - Gibbs ringing can be reduced with an apodization filter, e.g. "raw filter" (Siemens), "Image filter" (Philips). }\end{array}$ \\
\hline
\end{tabular}




\begin{tabular}{|c|c|c|}
\hline Purpose & $\begin{array}{l}\text { - Segmenting the SC and GM for measuring cord/WM/GM } \\
\text { CSA } \\
\text { - Registering to a template and accounting for GM shape } \\
\text { - Measuring SC and GM CSA }\end{array}$ & $\begin{array}{l}\text { - Computing MTR, MT-CSF and MTsat (requires T1w to } \\
\text { partially compensate for B1+ homogeneity and T1 } \\
\text { effects on the MTR }{ }^{106} \text { ) } \\
\text { - Detecting WM }{ }^{58} \text { and GM pathology (myelopathy) }\end{array}$ \\
\hline Pros & \multicolumn{2}{|c|}{$\begin{array}{l}\text { - Quantify SC neural tissue microstructural properties } \\
\text { - Sensitive to WM pathologies (e.g. degenerative demyelination, injury, edema, tumor) } \\
\text { - Longitudinal monitoring of patient-specific SC microstructure (i.e. disease progression) } \\
\text { - Detect origin of microstructural damage before non-reversible changes (e.g. T2w hyperintensities, appearance of } \\
\text { clinical symptoms) } \\
\text { - High in-plane axial resolution (good for atlas-based analysis of various WM tracts) } \\
\text { - The combined echoes provide high WM/GM contrast (depending on parameters). } \\
\text { - Fast } \\
\text { - Low SAR (except for the MT sequence) }\end{array}$} \\
\hline Cons & $\begin{array}{l}\text { - Sensitive to motion } \\
\text { - Sensitive to } B_{0} \text { inhomogeneities (signal drop-out due to intr } \\
\text { - Quantitative metrics sensitive to B1 (except for the ME-GR }\end{array}$ & $\begin{array}{l}\text { ra-voxel dephasing, can be mitigated using thinner slices) } \\
\text { E sequence) }\end{array}$ \\
\hline Parameters & $\begin{array}{l}\text { - Optimization: The parameters below result from a } \\
\text { consensus which arose from the "Gray Matter } \\
\text { Acquisition Challenge", which was organized during the } \\
\text { 5th Spinal Cord MRI workshop }{ }^{107} \text {. } \\
\text { - 2D vs. 3D: While 3D acquisitions are more SNR } \\
\text { efficient, we recommend using } 2 \mathrm{D} \text { acquisitions as they } \\
\text { produce "cleaner" images: no aliasing along the 2nd } \\
\text { phase encoding direction when using 3D, more } \\
\text { homogeneous B1+ profile than 3D acquisition, less } \\
\text { sensitive to motion. } \\
\text { - Spatial resolution: } 0.5 \times 0.5 \times 5 \text { mm } \\
\text { - Saturation band: adds a slight MT effect due to the } \\
\text { off-resonance pulse, which has the effect of slightly } \\
\text { increasing white/gray matter contrast. A corollary benefit } \\
\text { of this saturation band, positioned coronal and anteriorly } \\
\text { (Figure 8), is that it also removes signal from a region } \\
\text { prone to motion (swallowing and vessel pulsatility in the } \\
\text { neck). } \\
\text { - Monopolar vs. bipolar: This concerns the filling of } \\
\text { k-space across the different echoes. It is more } \\
\text { time-efficient to fill the k-space by alternating polarities } \\
\text { across echoes, however this leads to a slight inter-echo } \\
\text { shift caused by field inhomogeneities. For this reason we } \\
\text { recommend using monopolar encoding, with the } \\
\text { downside of slightly longer TE and TR. } \\
\text { - Multiecho combined: If individual echo images are } \\
\text { available; they should be combined during } \\
\text { post-processing for more transparency on the } \\
\text { aggregation method. MEDIC (Siemens), and MERGE }\end{array}$ & $\begin{array}{l}\text { - 2D vs. 3D: 3D is recommended because with some } \\
\text { vendors (incl. Siemens) the MT pulse is not selective, } \\
\text { therefore using a } 2 D \text { sequence will result in a different } \\
\text { MT effect across slices. } \\
\text { - Spatial resolution: } 0.9 \times 0.9 \times 5 \mathrm{~mm} \\
\text { - TR and Flip angle: optimized so as to minimize the } \\
\text { standard deviation of the MTR, given the limitations on } \\
\text { different systems (ex: SAR, TR, etc.). } \\
\text { - TE: minimized to reduce T2* relaxation. } \\
\text { - Saturation bands: not recommended because the } \\
\text { offset excitation creates an MT effect, which could vary } \\
\text { across vendors (different implementation). } \\
\text { - Fat saturation pulses: should not be used with a } \\
\text { quantitative MT protocol (MTR, MTsat, qMT, MTCSF, } \\
\text { etc.) because the off resonance pulse will create an } \\
\text { additional MT effect. } \\
\text { - Water excitation (using composite binomial pulses): } \\
\text { not recommended since the effect on the off-resonance } \\
\text { fat signal will impact the MT effect. } \\
\text { - MT frequency offset: higher frequency offset (e.g. } \\
\text { 4kHz) gives better WM/GM contrast and hence should } \\
\text { be used if GM segmentation is part of the analysis. } \\
\text { However, when using product sequences, the frequency } \\
\text { offset cannot be changed: Siemens and GE use } 1.2 \mathrm{kHz} \\
\text { while Philips uses } 1.1 \mathrm{kHz} \text {. } \\
\text { Multiecho combined: Although combining echoes } \\
\text { provides higher SNR, we don't always recommend it } \\
\text { because signal dropout at later echo times could bias }\end{array}$ \\
\hline
\end{tabular}




\begin{tabular}{l|l} 
(GE) automatically combine all echoes. The Philips & MT metrics. Also, on GE systems, it is not possible to \\
mFFE sequence outputs all the echoes with the option & use multiecho with the MT pulse. \\
to also output an "accumulated" image, which \\
corresponds to the sum of all echoes. Depending on the \\
version, the MEDIC sequence does not feature the \\
phase stabilization option (navigator-based phase \\
correction which minimizes ghosting), whereas the \\
FLASH does (this depends on the version, e.g.: VE11C \\
does not). \\
QC \\
- Check for blurry GM/WM edges, which could be caused by subject or cord motion. \\
- Check for ghosting artifacts (patterns of overlapped tissue on the cord) \\
- Fophasing. Workarounds include: reshimming, using thinner slices, reducing the TE (and/or number of echoes). \\
- For the MT acquisition: the 3D excitation does not have a sharp profile at the edges: we recommend discarding 2-3 \\
slices at each edge.
\end{tabular}

Table 4. Overview of parameters for the ME-GRE and the MT sequence. QC stands for "Quality

Control".

\section{DWI (2D axial)}

\begin{tabular}{|c|c|}
\hline Purpose & $\begin{array}{l}\text { - Computing DTI metrics (fractional anisotropy (FA), mean diffusivity (MD), radial diffusivity (RD), axial diffusivity (AD)) } \\
\text { that are sensitive to axonal damage, demyelination and degeneration }{ }^{108} \text {. }\end{array}$ \\
\hline Pros & $\begin{array}{l}\text { - Quantify SC neural tissue microstructural properties } \\
\text { - Sensitive to WM pathologies (e.g. degenerative demyelination, injury, edema, tumor) } \\
\text { - Longitudinal monitoring of patient-specific SC microstructure (i.e. disease progression) } \\
\text { - Detect origin of microstructural damage before non-reversible changes (e.g. T2w hyperintensities, appearance of } \\
\text { clinical symptoms) } \\
\text { - Short acquisition time }(<5 \mathrm{~min})\end{array}$ \\
\hline Cons & $\begin{array}{l}\text { - Sensitive to } B_{0} \text { inhomogeneities (EPI readout) } \\
\text { - DTI metrics are biased by SNR }{ }^{109}\end{array}$ \\
\hline Parameters & $\begin{array}{l}\text { 2D axial: for spinal tract-based metric quantifications (see section Slice orientation) } \\
\text { - Spatial resolution: } 0.9 \times 0.9 \times 5 \mathrm{~mm} \\
\text { - FOV: Reducing the FOV along the phase-encoding direction for EPI reduces susceptibility-related distortions. SC } \\
\text { geometry is favorable to such acquisition techniques. There are three main techniques for reducing the FOV: } \\
\text { - } 2 \text { DRF excitation: FOCUS }{ }^{110} \text { (GE, version DV25 and onwards), ZOOMit }{ }^{111} \text { (Siemens). Paid licence. Not available } \\
\text { on all versions. } \\
\text { - Cross-sectional RF excitations "Zoom Diffusion" }{ }^{112,113} \text { (Philips): available for software versions } 5.3 \text { and higher. } \\
\text { For older software, it is possible to use a research sequence which is equivalent to the product sequence. } \\
\text { - Outer volume suppression with sat bands: (available for all vendors). Note: because the saturation band } \\
\text { approach is not perfect (fat can be missed and aliased on top of the SC, as exemplified in Figure } 3 \mathbf{3} \text { in } \\
\text { [REF-DATA]), it is strongly recommended that it only be used if other options are not available. } \\
\text { - For more details about the pros/cons of each reduced FOV technique for the SC, the reader is referred to }{ }^{114} \text {. } \\
\text { - Contiguous slices } \\
\text { - Pros: can do tractography, greater statistical power }\end{array}$ \\
\hline
\end{tabular}


- Cons: reduced coverage (more slices can be added at the discretion of the researcher)

- B-value: single-shell at $800 \mathrm{~s} / \mathrm{mm}^{2}$

- A single-shell protocol was chosen due to time constraints. For more advanced diffusion models (e.g. NODDI, DBSI), additional shells can be added, potentially increasing TE/TR as the b-value is increased.

- If the SNR is too low (the SC is barely visible on diffusion-weighted images), the b-value could be lowered (e.g., $600-700 \mathrm{~s} / \mathrm{mm}^{2}$ ), thereby reducing TE and increasing the SNR. Note that increasing the number of repetitions is not helpful because in this low-SNR regime the noise distribution is more Rician-like, hence averaging of the magnitude signal results in an upward bias. On the other hand, if the SNR is sufficient, on scanners with strong gradients $(\geqslant 80 \mathrm{mT} / \mathrm{m})$ it is recommended to set an additional b-value shell $\left(2000-3000 \mathrm{~s} / \mathrm{mm}^{2}\right.$ or higher) to provide better sensitivity to diffusion-based contrast (to see demyelination/degeneration) and enable the use of more advanced diffusion models. For any multi-shell acquisition, using a fixed TE across shells is recommended.

- Users should note that a low b-value $\left(800 \mathrm{~s} / \mathrm{mm}^{2}\right)$ may not detect complex fiber geometry for tractography applications (e.g., crossing, fanning). The b-value and number of diffusion directions can be modified to fit researchers' needs.

- Number of directions: $30-32$ with $5 b=0$ images (acquired at the beginning or interspersed ${ }^{127}$ ). $b=0$ should have the same TE as the DWI data.

- TR and cardiac gating: It is recommended to acquire EPI data during the quiescent phase of the cardiac-related SC motion ${ }^{115}$. The quiescent phase of the SC lasts for about 500 ms within a cardiac cycle ${ }^{115}$. On the Siemens platform, we added a concatenation to break down the volume acquisition and only acquire $\sim 3$ slices during the quiescent phase of the cord. On the Philips platform, 1 or 2 slices are acquired per beat (depending on the heart rate). We suggest using a pulse oximeter instead of an ECG (it has adequate precision and is less cumbersome to use). The trigger delay is subject-dependent ${ }^{115}$ and its definition depends on the platform and sequence parameters ${ }^{128}$, making it difficult to provide an optimal number that fits all subjects and platforms. For convenience, we set the delay to the minimum value, but researchers can optimize this value accordingly.

- TE: should be minimum (to maximize SNR).

- Echo spacing (controlled with the bandwidth): should be minimum (to minimize distortions).

- Diffusion gradient mode: monopolar ${ }^{129}$ (if available) to ensure lower TE. If needed, bipolar mode can be used to minimize eddy current-related distortions.

- Dynamic Stabilization: Frequency stabilization (Siemens), Dynamic Stabilization (Philips), Real Time Field Adjustment (GE): These options help reduce artifacts related to changes in the magnetic field over time.

- Phase Encoding: P-A instead of A-P so that (i) susceptibility distortions have the effect of "stretching" instead of "compressing" the SC (no information loss) and (ii) in case of poor fat saturation, posterior neck fat will be aliased outside of the FOV.

- Shimming box (+ advanced shimming) should be carefully positioned around the SC. See Figure 8.

- Acceleration (GRAPPA/SENSE): We recommend no in-plane acceleration because a small matrix size (96×96) combined with a reduced FOV ( $60 \%$ reduction) and partial Fourier $(7 / 8)$, leaves too few phase-encoding lines for reliable image reconstruction. Besides, in-plane acceleration reduces SNR by factor sqrt(R), where $R$ is the in-plane acceleration factor.

127 Possible by editing "DiffusionVectors.txt" (on Siemens) or "dti_vectors_input.txt" (on Philips) or "tensor.dat" (on GE), but probably only sensible for research sites.

${ }^{128}$ For example, the SPAIR fat saturation on the Siemens platform adds $\sim 83 \mathrm{~ms}$ before each excitation, while on the Philips platform the minimum delay is about $110 \mathrm{~ms}$ (used by the SPIR fat sat pulse and the outer volume suppression pulses).

${ }^{129}$ On older Siemens platforms (e.g. VB17), the ep2d_diff product sequence is bipolar, however research or work-in-progress sequences exist, such as WIP511 (monopolar option, with polarity alternation, freq stab and skewed fat sat). 


\begin{tabular}{|c|c|}
\hline & $\begin{array}{l}\text { - Simultaneous multi-slice: While this technique is gaining popularity for reducing the overall acquisition time by } \\
\text { exciting several slices at the same time, thereby reducing the volume TR, we don't suggest its use here because the } \\
\text { number of slices ( } n=15 \text { ) and their distance is small, which hampers the acceleration performance and further } \\
\text { reducing the TR would hamper longitudinal relaxation. } \\
\text { - Fat suppression: } 2 \mathrm{DRF} \text { protocols use water excitation while the Philips Zoom sequence employs a SPIR fat } \\
\text { saturation pulse to minimize fat contribution. If insufficient, skewed techniques }{ }^{116} \text { could be tried. } \\
\text { - Partial Fourier: } 75 \% \text { k-space to mitigate phase errors, while still being able to reduce TE. A larger k-space window } \\
\text { also minimizes the risk of a total drop-out that can happen when the peak of an echo moves entirely outside the } \\
\text { readout window. } \\
\text { - Fieldmap (blip-up/down): Given the difficulty of acquiring a robust fieldmap and correcting for susceptibility-related } \\
\text { distortions using a blip-up/down sequence in the SC (partly because the manifestation of artifacts in the up/down } \\
\text { directions might be slightly different, due to e.g., CSF presence, } \mathrm{B}_{0} \text { field differences between up/down because of } \\
\text { respiratory-related } \mathrm{B}_{0} \text { variation), we do not recommend that these be acquired. Any type of correction might } \\
\text { introduce more artifacts if misused. Instead, we suggest acquiring data with minimal distortions in the first place (by } \\
\text { minimizing the echo spacing, optimizing shimming, etc.) and correcting residual distortions by registering the DWI } \\
\text { data on to a structural scan (e.g. } b=0 \text { on the T2 space). For more details, see this forum post }{ }^{130} \text {. }\end{array}$ \\
\hline QC & $\begin{array}{l}\text { - If saturation bands are used: Look for poorly saturated signals in the saturation band region by prescribing full FOV } \\
\text { and looking at the area where saturation bands are located. If the signal outside the FOV is too high, causing it to } \\
\text { alias over the SC, try to increase the number of phase encode lines (this will cause slightly more distortions) or to } \\
\text { unselect coil elements if they are not necessary (e.g., switching off the anterior neck element). } \\
\text { - Poor fat saturation causing fat to overlay on the SC (see Figure } 3 \mathrm{f} \text { in [REF-DATA]): The cause is likely related to } \\
\text { poor shimming. In this case, try to move the table, re-shim, and/or try other fat saturation methods (e.g. } \\
\text { frequency-selective, inversion-recovery). } \\
\text { - Automatic outlier slice/frame detection (to be excluded from DTI modeling) using a machine learning algorithm }{ }^{117} \text {. }\end{array}$ \\
\hline
\end{tabular}

${ }^{130}$ http://forum.spinalcordmri.org/t/how-to-correct-for-distortions-in-spinal-cord-diffusion-mri-data/326 


\title{
Procedure
}

\section{Equipment setup. TIMING: 2 min}

\author{
1. Install coil
}

Select the coil depending on your vendor and application (Table 6).

\section{Cervical}

\begin{tabular}{|c|c|c|c|}
\hline \multirow{3}{*}{ GE } & HD/HDxt & 8-ch Cervical Thoracic Lumbar (CTL) array & 8-ch CTL array \\
\hline & PETMR & 19-ch Head Neck Unit (HNU) array & 14-ch Central Molecular imaging Array (CMA) \\
\hline & MR750w & 16-ch Head Neck Spine (HNS) array & $\begin{array}{l}\text { 48-ch Geometry Embracing Method Phased Array } \\
\text { (GEMPA) }\end{array}$ \\
\hline \multirow[t]{2}{*}{ Philips } & Achieva & 16-ch head/neck/neurovascular & 15-ch posterior spine $\left.{ }^{* *}\right)$ \\
\hline & Ingenia(*) & 32-ch head coil & 12-ch posterior array $\left({ }^{* *}\right)$ \\
\hline \multirow{5}{*}{ Siemens } & $\begin{array}{l}\text { Trio } \\
\text { Verio }\end{array}$ & $\begin{array}{c}\text { 12-ch brain }+4 \text {-ch neck array }+ \text { spine } \\
\text { array }\left({ }^{* *}\right)\end{array}$ & \multirow{5}{*}{ Spine array $\left.{ }^{* *}\right)$} \\
\hline & Skyra & & \\
\hline & D & 64-ch head/neck & \\
\hline & & 20 -ch head/neck + spine array $\left({ }^{* *}\right)$ & \\
\hline & Vida & & \\
\hline
\end{tabular}

Table 6. Recommended receive coils for SC imaging for GE, Philips and Siemens systems. The coils in red font are used by default in the spine generic protocol. (*): (i) posterior spine coil could also be used depending on coverage, (ii) for thoracic/lumbar SC imaging; anterior coil could be used to improve image quality in sequences with anterior-posterior phase-encoding. $\left(^{* *}\right)$ : The relevant elements of the spine array are to be selected depending on the region to cover. When using "auto select" (Siemens) or "SmartSelect" (Philips), elements will be automatically selected based on the slice positioning. It is advised to use it. This table is subject to changes with the evolution of the market.

\section{Subject preparation - TIMING: 5-10 min}

\section{Positioning and immobilization strategies}

Careful positioning of the subject can have an impact on the overall image quality. In general, it is desirable to have the cervical cord almost straight so that axial slices are orthogonal to the cord centerline; this minimizes partial volume effects with the surrounding cerebrospinal fluid 
(CSF). Reducing neck curvature also helps to improve field homogeneity because the shim volume (i.e., the 3D box centered over the region of interest where the MR system computes the optimal shim coefficients) is less likely to contain air-tissue interfaces. To minimize cervical lordosis, ask the subject to tilt their head slightly towards their chest. Placing some cushions below the head can help, as illustrated in Figure 3. However, subjects should not be too uncomfortable and still be able to swallow in a way that minimizes their motion. For thoraco-lumbar acquisitions, leg support helps minimize lumbar lordosis and provides more comfort for the subject.

It is also important to verify that the subject is aligned in the left-right direction, ensuring alignment of the spine with the sagittal plane whenever possible.
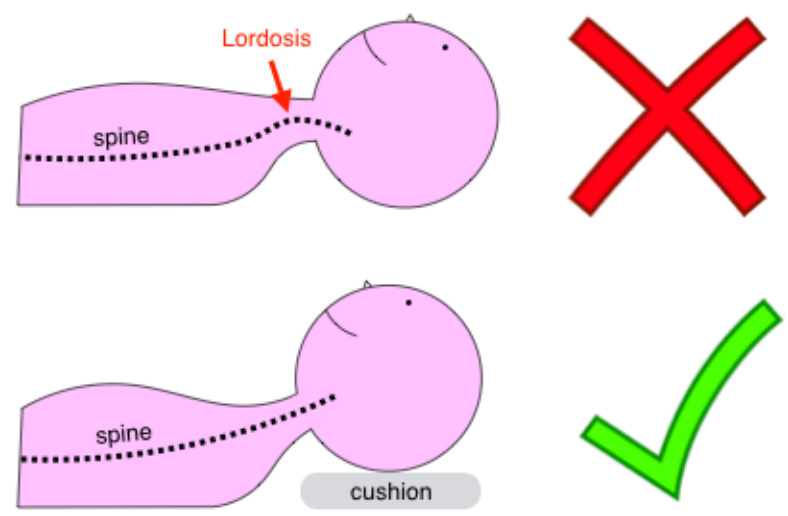

Figure 3. Suggested subject positioning: Use a cushion to minimize cervical lordosis (bottom panel)

A cervical collar is effective for reducing motion ${ }^{118}$. However, some disadvantages include that it may: (i) not fit in the coil (e.g., 64ch), (ii) increase the natural cervical cord lordosis, (iii) create discomfort for some subjects.

Custom tight-fitting helmets, such as the CaseForge (https://caseforge.co/), are personalized helmets that fit perfectly in specific coil models. Their use has been shown to lead to highly reproducible positioning throughout longitudinal studies ${ }^{119}$, however, they require customization and, hence, are not a viable solution for large-scale multi-center studies.

Padding/clamping the subject's head tightly with cushions to avoid head motion is what we recommend here for the spine generic protocol. While this has the merit of not requiring additional purchases (e.g. collar or specialized immobilization apparatus), this setup is not easily reproducible and depends on the MR technician. Also, it does not ensure that subjects are always positioned in the same way for longitudinal experiments. Therefore, it is important that researchers specify the type of cushions used and ideally take a picture showing how to position those cushions while the subject is in the coil. 


\section{Clear communication with the subject}

It is important for the subject to understand that their neck/spine will be imaged and that if they move, image quality may be severely compromised. Mimic how not to swallow by exaggerating head and swallowing motions. Asking subjects not to swallow at all can sometimes lead to more motion due to the swallowing reflex that is triggered once a large volume of saliva is accumulated. This can also pose a choking risk, given that subjects are in a supine position. As a compromise, notify the subject when they can swallow between scans.

Respiration has a detrimental impact on $\mathrm{B}_{0}$ dynamic variation ${ }^{70}$, causing ghosting on gradient echo data and pixel displacement on EPI sequences. Breathing pattern is important and subjects should breathe normally, avoiding deep breaths.

\section{Pulse Oximeter}

Install the pulse oximeter which will be used for cardiac gating on the DWI scan.

\section{Positioning the Isocenter (laser marking)}

If you are doing brain and cervical cord imaging, mark the isocenter (laser) right below the nose (Figure 4). This will ensure that the localizer will cover the desired region. Note that for all other sequences, the table will move so that the center of the FOV is acquired at the scanner's isocenter (to ensure maximal gradient linearity).

For thoracic/lumbar applications, set isocenter around the region of interest.

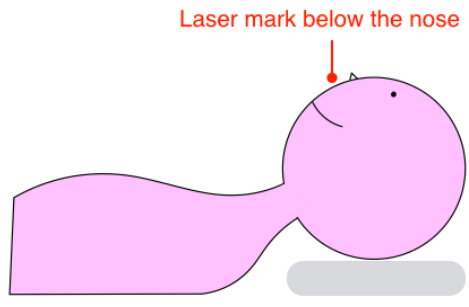

Figure 4. Recommended location for isocenter position.

Image Acquisition • TIMING: 20-30 min

\section{T1w Scan}

Adjust the FOV so that it includes the whole head, as shown in Figure 5 and acquire the T1w scan.

$\triangle$ CRITICAL Before starting the acquisition, make sure the coil elements are properly selected. If you are using a coil which corresponds to the saved protocol (in red in Table 6), the correct 
elements should be automatically selected. If you are not using a default coil, or if you are acquiring in the thoraco-lumbar region, then you will need to select the elements corresponding to the FOV. In some vendors and platforms the elements will be automatically selected depending on the location and size of the FOV (mode "auto select" or "SmartSelect"), but regardless it is always important to double check.

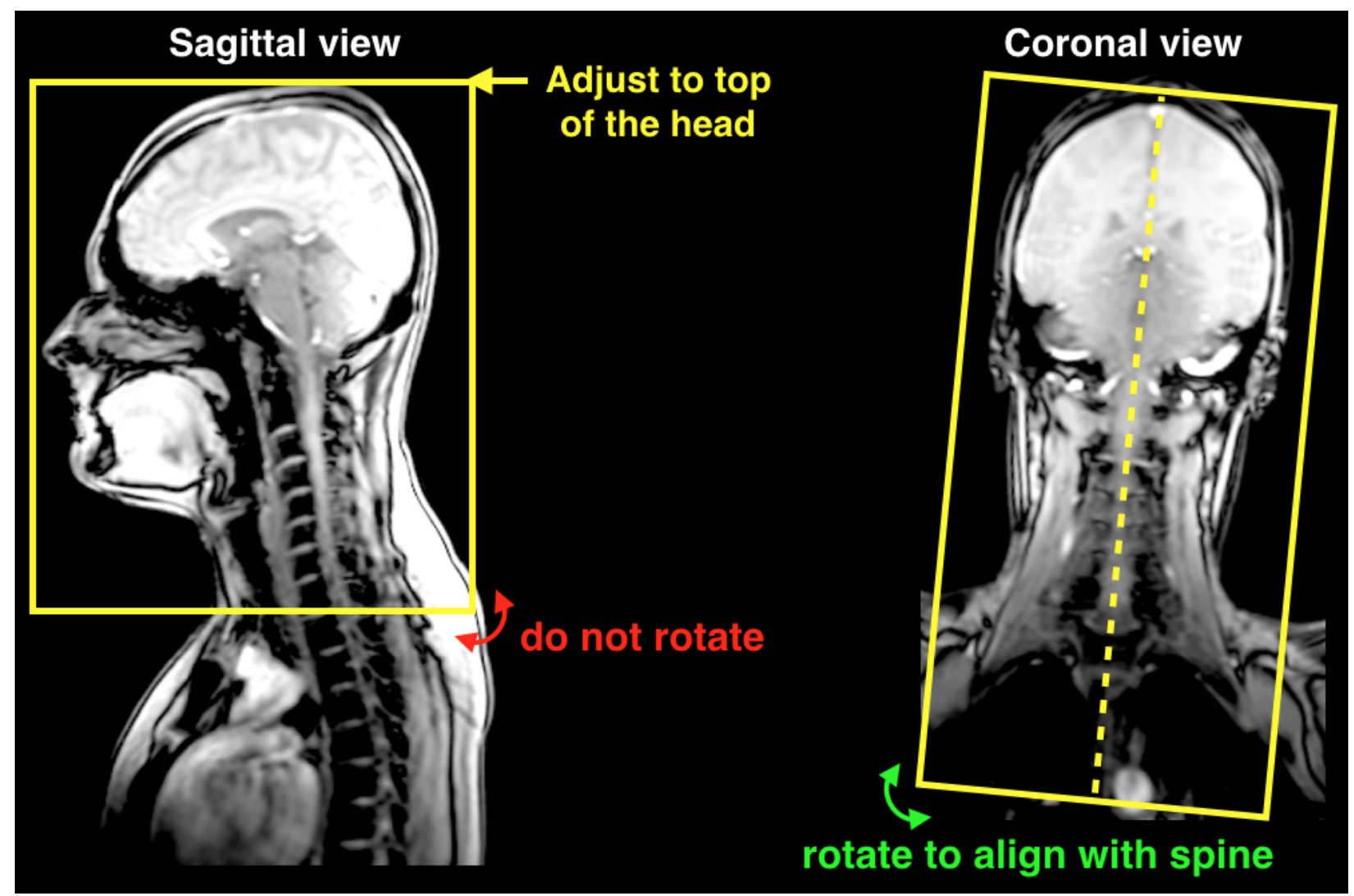

Figure 5. Positioning of FOV for T1w scans.

\section{! CAUTION}

GE users: To avoid confusion with regards to the slice orientation, the protocol is saved as "sagittal". Please click on "oblique" to be able to rotate the slice in the coronal plane.

Optional: To have the images reconstructed at the proper matrix size, click on "Save $\mathrm{Rx} \rightarrow$ "Scan", then click on "Research" $\rightarrow$ "Download". Then Click on "Research" $\rightarrow$ "Display CVs". Then, modify the following CVs accordingly ${ }^{131}$ :
- rhimsize=320
- rhrcxres $=320$
- rhrcyres $=256$

\footnotetext{
${ }^{131}$ You can check on the console if the field was modified appropriately, by looking at the "image header", after reconstruction. You should get: $(0 x 0028,0 \times 0010)=192 ;(0 x 0028,0 \times 0030)=111$
} 


\section{? Troubleshooting}

\section{T2w Scan}

Center the FOV at C3-C4 as shown in Figure 6. Align along the spine (see coronal view) and acquire the T2w scan.

$\triangle$ CRITICAL Make sure the proper coil elements are selected.

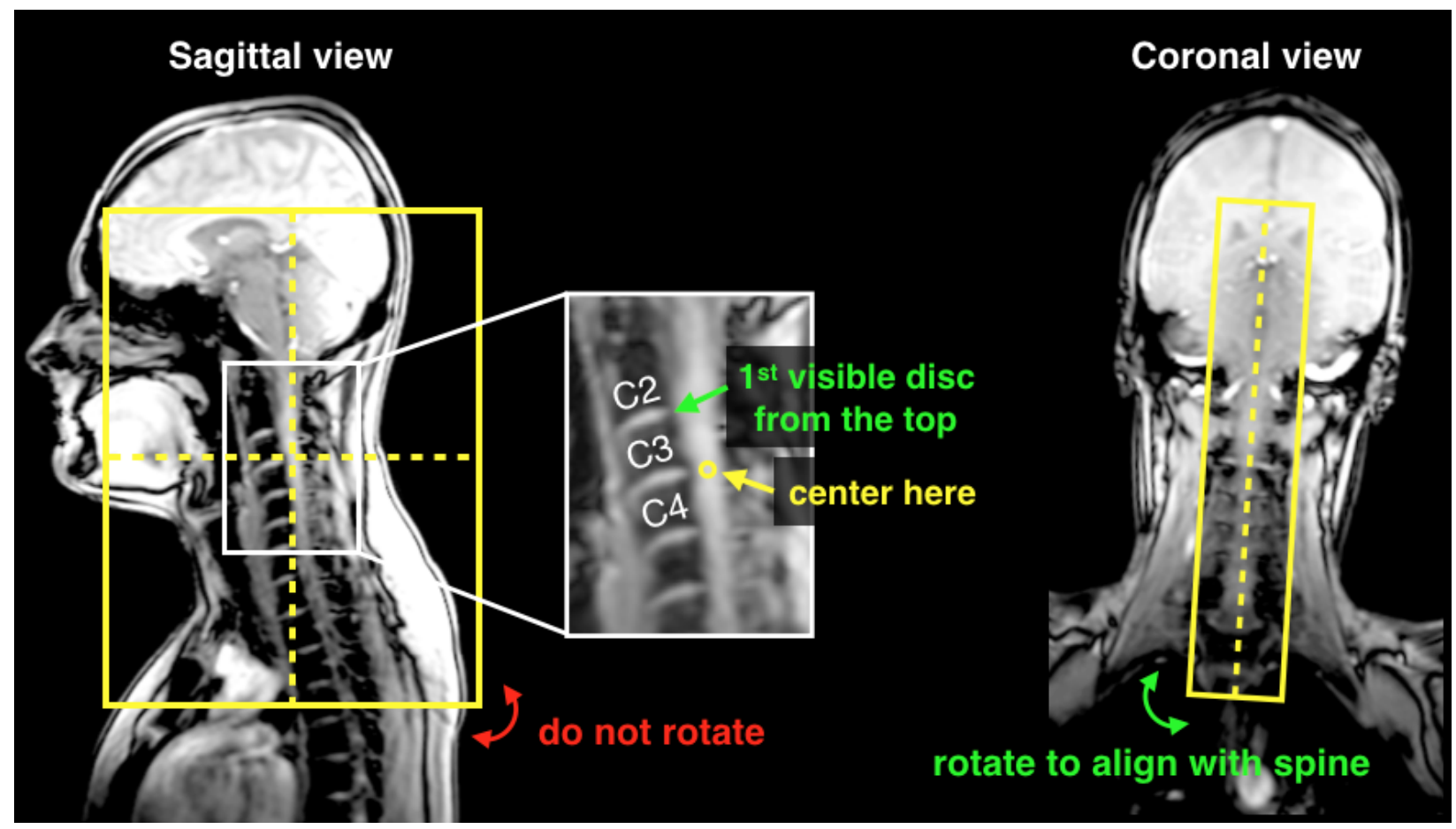

Figure 6. Positioning of FOV for T2w scans.

\section{! CAUTION}

GE users: To avoid confusion with regards to the slice orientation, the protocol is saved as "sagittal". Please click on "oblique" to be able to rotate the slice in the coronal plane.

Optional: To have the images reconstructed at the proper matrix size, click on "Save Rx $\rightarrow$ "Scan", then click on "Research" $\rightarrow$ "Download". Then Click on "Research" $\rightarrow$ "Display CVs". Then, modify the following CVs accordingly:
- rhimsize=320
- rhrcxres=256
- rhrcyres=256

\section{? Troubleshooting}




\section{GRE-ME Scan}

The FOV center and orientation should be the same as for the DWI scan. Normally, if you imported the full protocol, the FOV should be copied automatically from the DWI scan. If not, please do "copy parameters" (center of FOV and orientation).

$\triangle$ CRITICAL Make sure the proper coil elements are selected.

Shimming: Adjust shim box so that it follows the spine as closely as possible (see Figure 7).

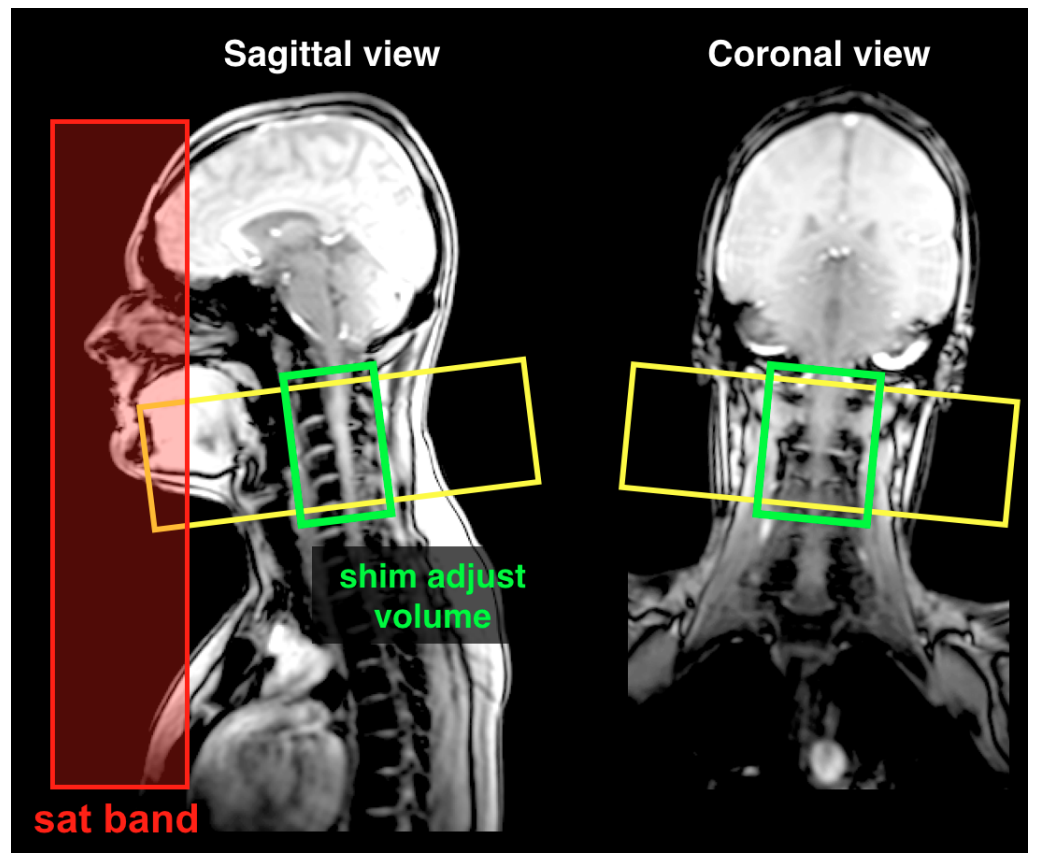

Figure 7. Positioning of the FOV, shim box and saturation bands for the GRE-ME scan. Siemens and GE users: the saturation band is already automatically positioned. Philips users: the saturation bands are "invisible" on this sequence, but are nevertheless applied.

\section{! CAUTION}

GE users: To avoid confusion with regards to the slice orientation, the protocol is saved as "axial". Please click on "oblique" to be able to rotate the slice in the sagittal and coronal planes.

Optional: To have the images reconstructed at the proper matrix size, click on "Save Rx $\rightarrow$ "Scan", then click on "Research" $\rightarrow$ "Download". Then Click on "Research" $\rightarrow$ "Display CVs". Then, modify the following CVs accordingly ${ }^{132}$ :

\footnotetext{
132 You can check on the console if the field was modified appropriately, by looking at the "image header",
} after reconstruction. You should get: $(0 \times 0028,0 \times 0030)=0.510 .5$ 
- rhimsize=448

- rhrcxres $=224$

- rhrcyres $=224$

\section{? Troubleshooting}

\section{GRE-MT1 / MT0 / T1w Scans}

The FOV center and orientation should be the same as for the DWI scan. Normally, if you imported the full protocol, the FOV should be copied automatically from the DWI scan. If not, please do "copy parameters" (center of FOV and orientation).

$\triangle$ CRITICAL Make sure the proper coil elements are selected.

Shimming: Use "auto" mode.

$\triangle$ CRITICAL If you get a SAR limitation on the MT scan, then increase the TR to the minimum suggested (e.g., going from $35 \mathrm{~ms}$ to $36 \mathrm{~ms}$ ). In that case, it is very important that you also change the TR on the GRE-MTO sequence (TR should be the same on the MT1 and MT0 scans).

\section{! CAUTION}

GE users: To avoid confusion with regards to the slice orientation, the protocol is saved as "axial". Please click on "oblique" to be able to rotate the slice in the sagittal and coronal planes.

To match the RF frequency of other vendors, modify the CV off_rfmt

Optional: To have the images reconstructed at the proper matrix size, click on "Save Rx $\rightarrow$ "Scan", then click on "Research" $\rightarrow$ "Download". Then Click on "Research" $\rightarrow$ "Display CVs".

Then, modify the following CVs accordingly:

- rhimsize=192

- rhrcxres=172

- rhrcyres=172

\section{? Troubleshooting}




\section{DWI Scan}

The following steps are illustrated in Figure 8.

1) Center the FOV in the cord at the level of $\mathrm{C} 3 / \mathrm{C} 4$ disc.

2) Rotate the FOV such that slices are orthogonal to the spinal cord, in both the sagittal and coronal planes.

3) Adjust the shim volume such that it covers the FOV, in both the sagittal and coronal planes (green box).

4) a. (prefered) If you have Zoomlt (Siemens), ZOOM (Philips) or FOCUS (GE).

b. If you don't have the license, use saturation bands for aliasing suppression.

$\triangle$ CRITICAL Make sure the proper coil elements are selected.

\section{! CAUTION}

GE users: Change the "axial" to "oblique" to be able to rotate the FOV. When tilting the slice, the TE might increase by a few ms. If you wish to use the same TE throughout an entire study, we suggest you try tilting the FOV in the coronal and sagittal plane, and report what the minimum TE is. The more you tilt, the bigger the TE will be (hence lower SNR) but the more conservative you will be in keeping a fixed TE throughout the entire study.

$\triangle$ CRITICAL Phase-encode should be A-P.

\section{! CAUTION}

GE users: Click on "shim volume" and then center on the spinal cord. If you cannot modify the size of the shim box, don't worry. 


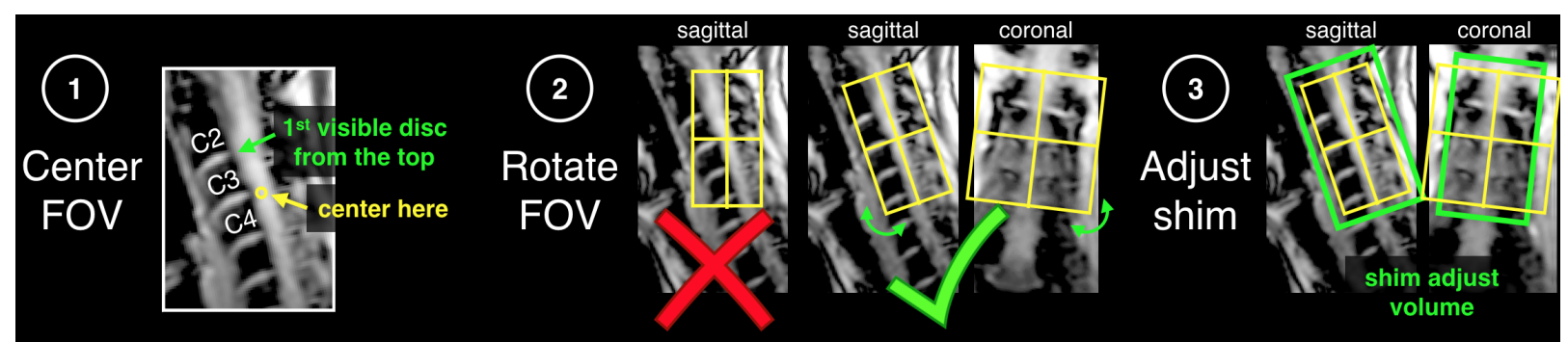

4a With Zoomlt (Siemens), ZOOM (Philips) or FOCUS (GE)
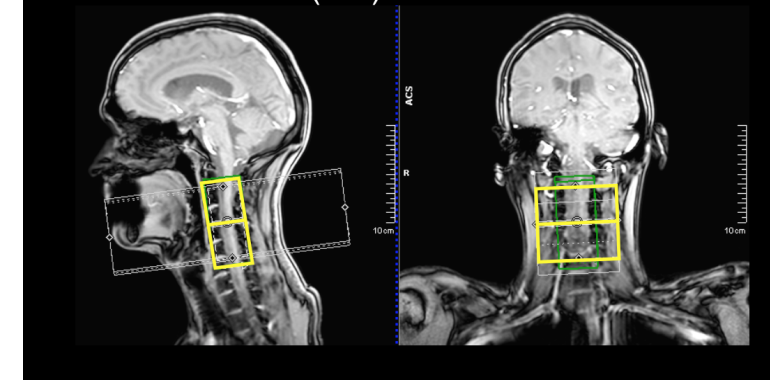

(4b) Without special reduced-FOV sequence, 4b use saturation bands to avoid aliasing.
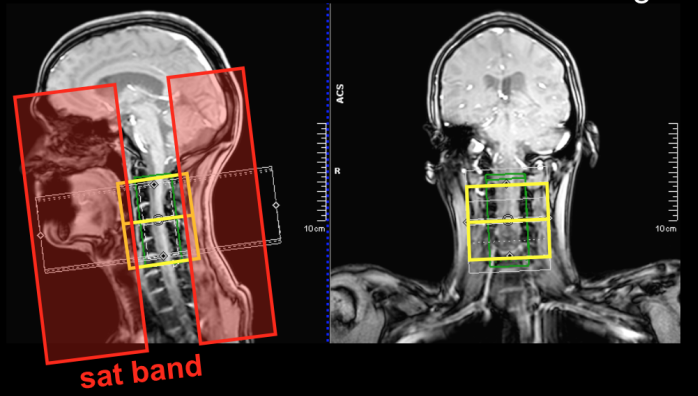

Figure 8. Positioning of FOV, shim box and saturation bands for the DWI scan.

$\triangle$ CRITICAL Before starting the acquisition, make sure the PulseOx trigger is working. It should look like what is shown in Figure 9:

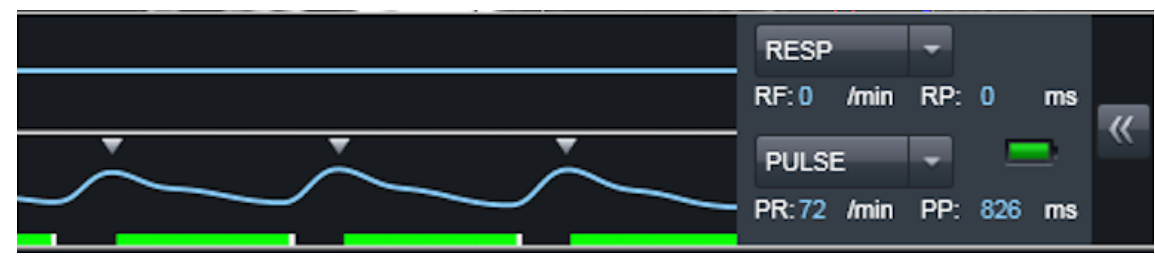

Figure 9. Example of pulse oximeter trace on a Siemens scanner for triggered acquisition (small triangles).

\section{! CAUTION}

GE users: The current protocol (v2) is saved without pulseOx gating. We recommend you add it (select "PG gating").

Optional: To have the images reconstructed at the proper matrix size, click on "Save $\mathrm{Rx} \rightarrow$ "Scan", then click on "Research" $\rightarrow$ "Download". Then Click on "Research" $\rightarrow$ "Display CVs". Then, modify the following CVs accordingly:
- rhimsize=96
- rhrcxres $=86$
- rhrcyres $=43$ 
? Troubleshooting 


\section{Troubleshooting}

The following is a list of common image artifacts that could arise due to errors on the part of the scan operator or due to subject motion.

$\triangle$ CRITICAL It is extremely important that you check each image right after its acquisition, not wait until the end of the imaging session. For example, if you notice that the wrong coil was used, fix the problem for the rest of the images (and reacquire the image if there is still time). Or if you spot excessive subject motion, talk to the subject before acquiring the next image.

\section{Wrong coil selection (step 1)}

For each region in the FOV, the proper coil needs to be selected (Table 6). For example, if you are planning to cover the head and neck region, then the head/neck coil should be used. In addition, for each sequence, the proper coil elements need to be selected, otherwise it could lead to insufficient signal in some parts of the image as illustrated in Figure 10. If you notice such artifacts in the image, make sure to check those coil parameters.

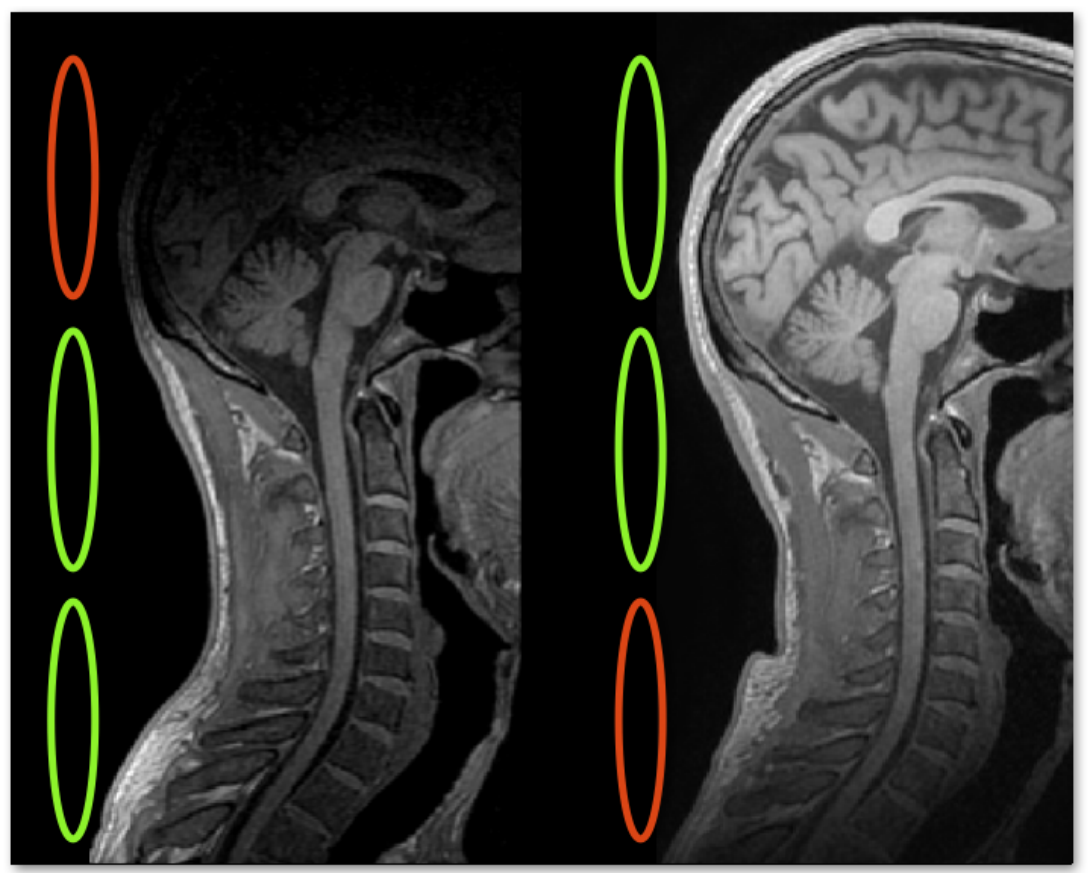

Figure 10. Illustration of the effect of not using the appropriate coil and/or not selecting the appropriate elements. On the left, the head coil elements (shown in red) were inactive during acquisition of the T1w scan (step 6), resulting in insufficient signal in the head region. On the right, the elements corresponding to the lower c-spine were inactive, resulting in low signal in this region. 


\section{Improper subject positioning: Excessive lordosis (step 2)}

Proper subject positioning is important both for the subject's comfort (which has an indirect positive impact on image quality) and for the reduction of some artifacts. For example, excessive lordosis can create more pronounced SC motion (Figure 11).

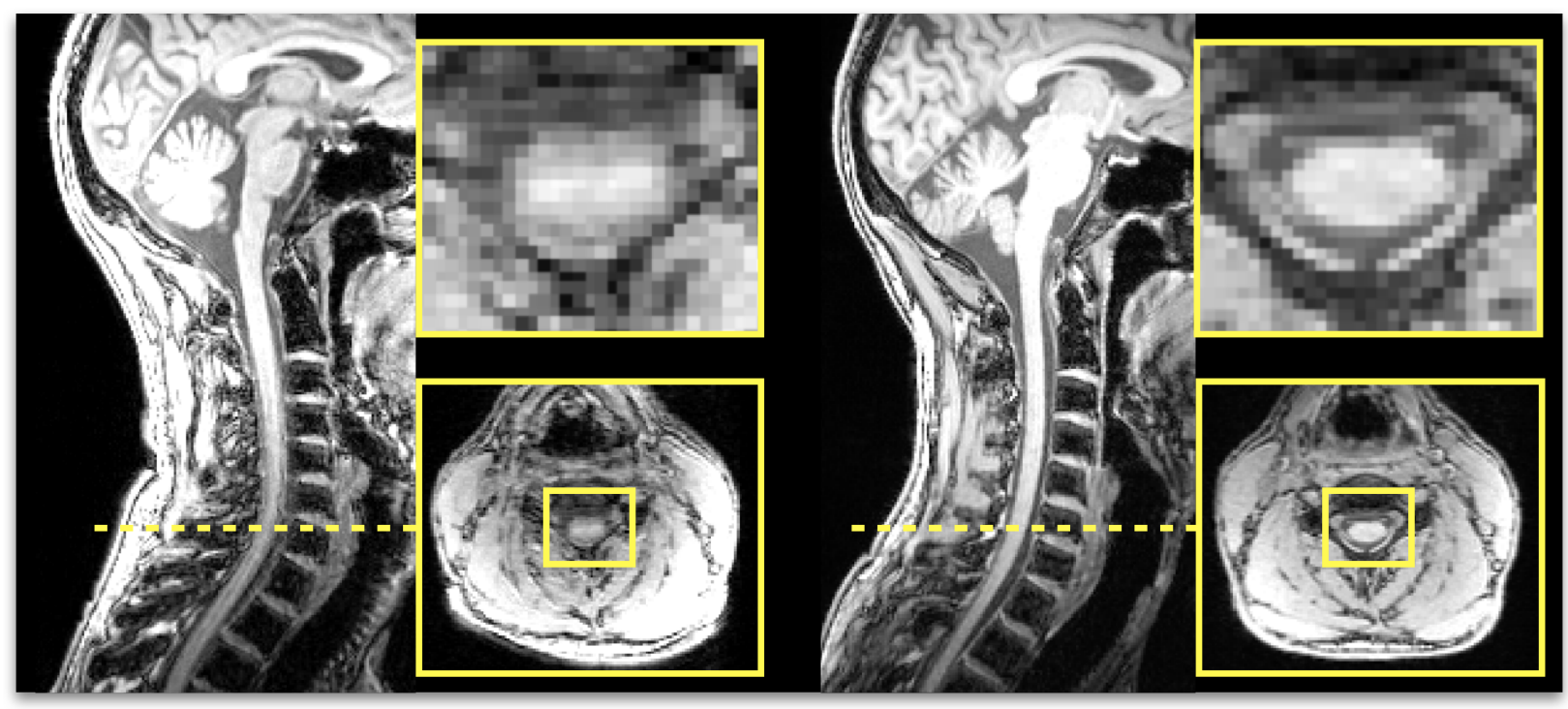

Figure 11. T1w MPRAGE taken in the same subject (from the single-subject database) at two different sites on a Siemens Prisma system. The slightly larger cervical lordosis on the left likely induced more pronounced CSF flow and SC motion resulting in the artifact shown in the axial view. 


\section{Improper subject/FOV positioning: Alignment in sagittal plane (step 2)}

Another example of bad subject positioning is when the medial plane of the spine and head is not aligned with the MRI bore. This could lead to the subject's discomfort and misalignment of the images if the FOV is not properly rotated about the antero-posterior axis (Figure 12).
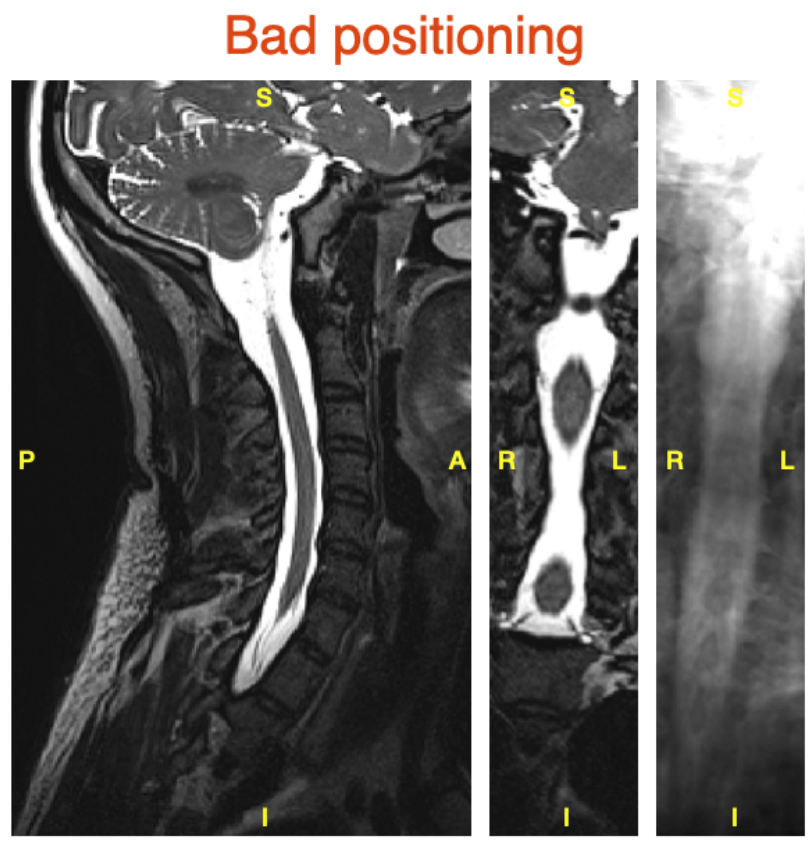

Good positioning
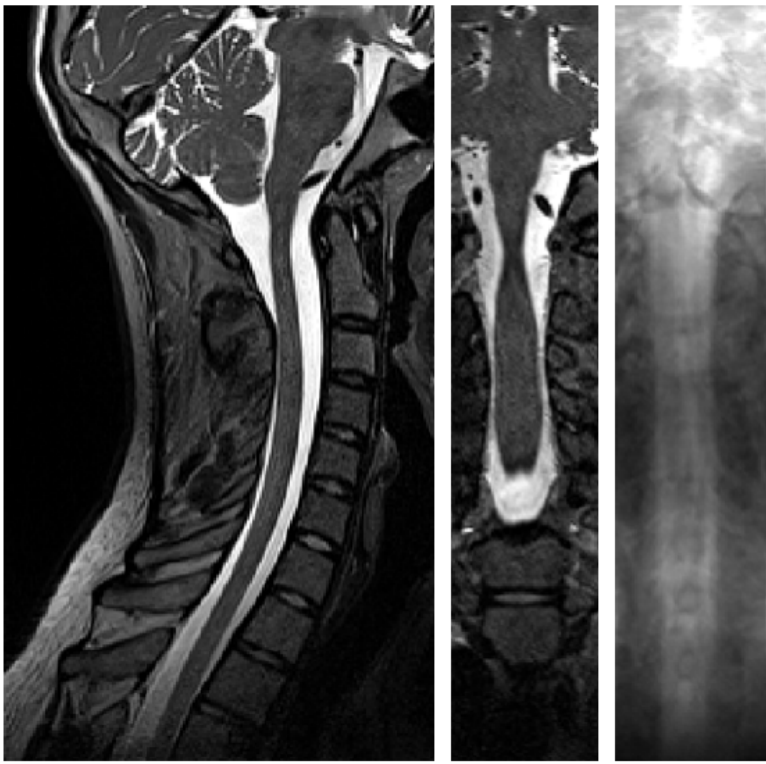

Figure 12. T2w images illustrating a subject improperly aligned in the scanner (left). The effect could have been mitigated by rotating the FOV to align it with the medial plane (right). Left and right panels show views of the sagittal plane, coronal plane and a coronal plane with all slices averaged to highlight the orientation of the spine. 


\section{Incorrect acquisition parameter: poor CSF signal recovery (step 7)}

Changes to the acquisition parameters can result in undesired artifacts, such as the one illustrated in Figure 13. In this example, the flip angle was increased, causing the CSF signal to not recover fully (hypointense signal). Other changes in sequence parameters (TR, TE, matrix size, etc.) could also lead to undesired artifacts or biases in the computed qMRI metrics.

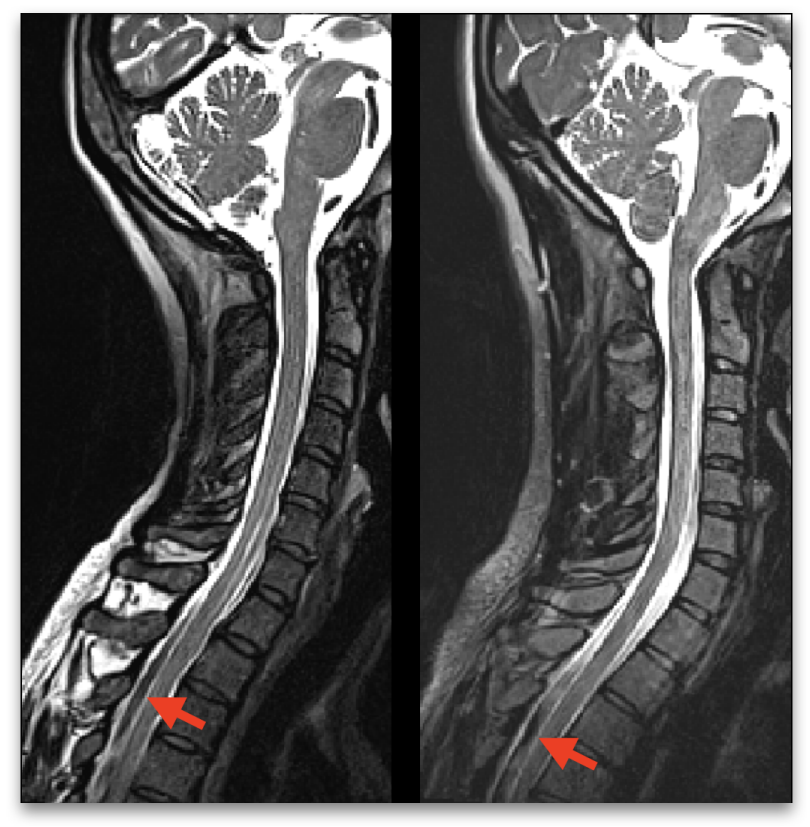

Figure 13. T2w scans showing signal drops in the CSF likely due to a poorly-recovered CSF signal combined with flow effects. These two subjects were acquired with $F A=180^{\circ}$ instead of the recommended $120^{\circ}$, which likely explained the presence of those artifacts. A TR shorter than the recommended value would produce a similar artifact, due to insufficient $T 1$ recovery in the CSF. 


\section{Motion artifact (steps 6-10)}

Subject motion can negatively affect all sequences, with some sequences being particularly sensitive: T1w (step 6), GRE-ME (step 8) and GRE-MT0/MT1/T1w (step 9). See for example Figure 14. When you observe excessive motion artifacts after an image is reconstructed, it is a good idea to talk to the subject, asking them to not move for the rest of the imaging session. If there is still time before the end of the imaging session, it is recommended to re-acquire the problematic image(s).
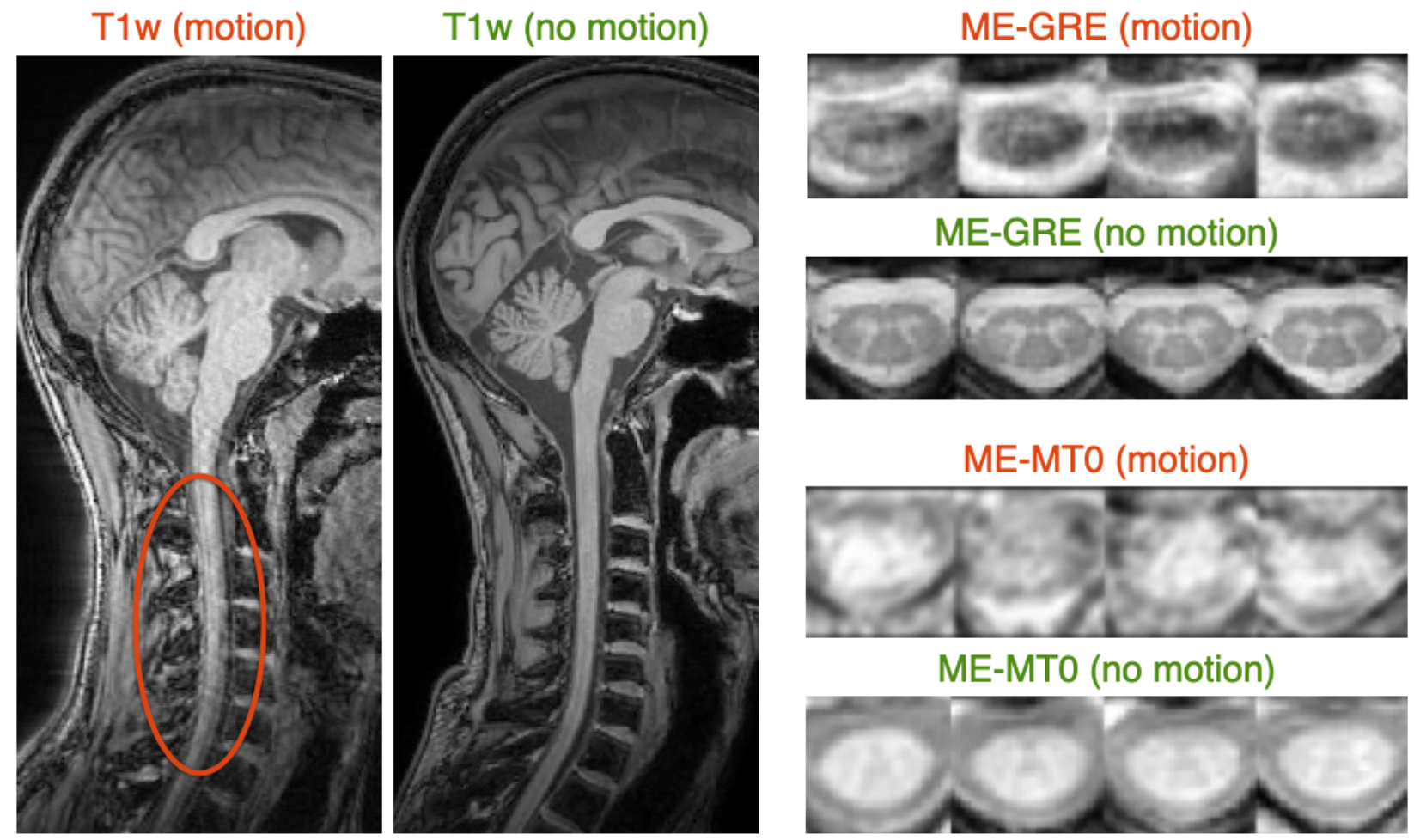

Figure 14. Illustration of the effect of subject motion on T1w (step 6), ME-GRE (step 8) (zoomed view) and ME-MTO scans (step 9) (zoomed view). 


\section{Wrong FOV placement (steps 6-10)}

It is important to follow the prescribed FOV placement, failure to do so could result in variable coverage across the studied population, and be a source of inconsistencies and biases. Figure 15 shows an example of wrong FOV placement for a GRE-MT scan.
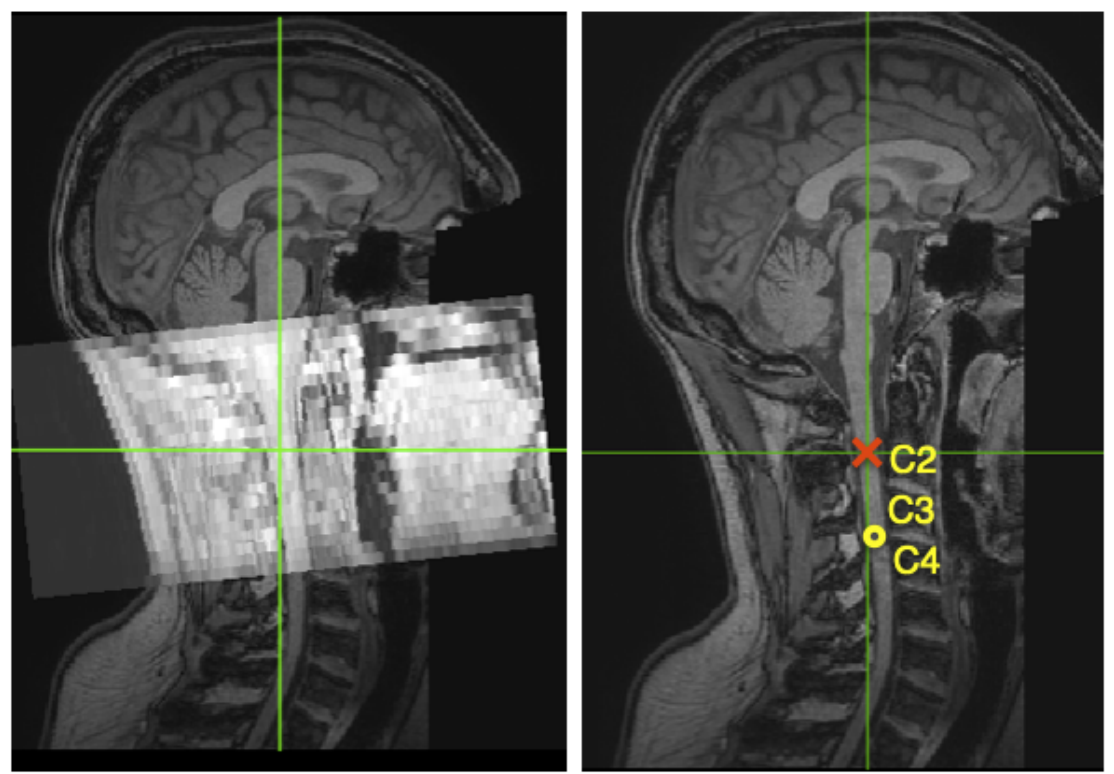

Figure 15. The left panel shows a GRE-MT image overlaid on a T1w image. The green cross is at the center of the FOV of the GRE-MT image. The right panel shows the T1w image without the overlay. There, we can see that the GRE-MT FOV is centered at the C2 vertebrae, whereas the requested FOV center was at the level of the C3-C4 intervertebral disc (step 9). 


\section{Image artifacts on DWI scans (step 10)}

The DWI scan is based on an EPI sequence, which is prone to susceptibility artifacts manifesting as image distortions. Other effects can lead to artifacts when using this sequence, including poor fat saturation and excessive subject or pulsatile motion. Figure 16 illustrates some of the artifacts you might encounter.
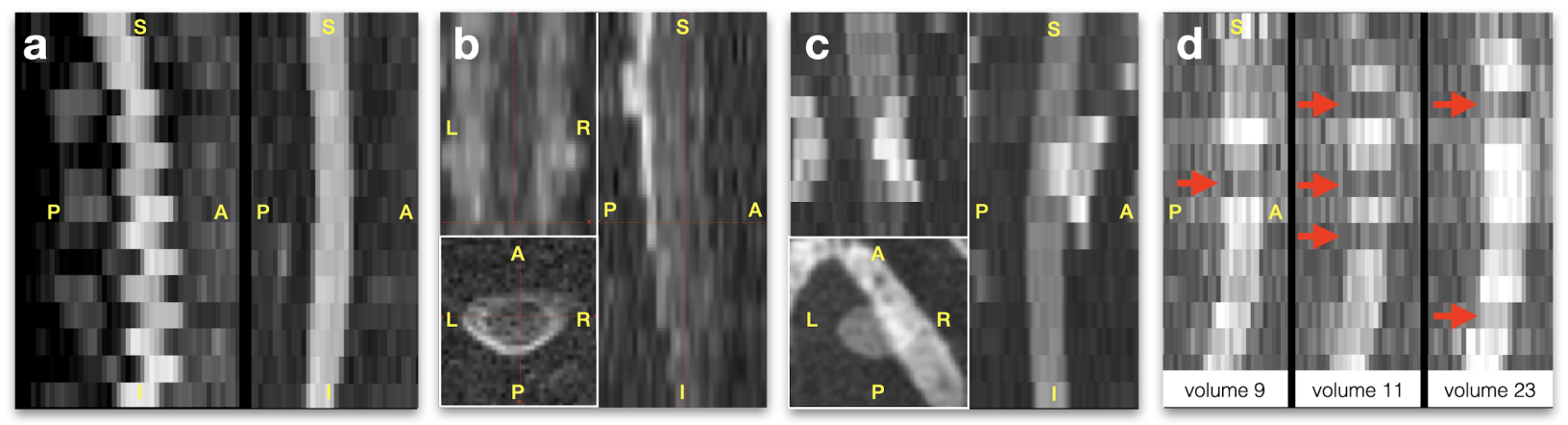

Figure 16. (a) Mean DWI scan from a Philips site (left) with a concatenated acquisition wherein odd slices are acquired during the first half of the entire acquisition (spanning all b-vectors) and the even slices are acquired during the second half. In the event of subject motion between those two acquisition sub-sets, apparent motion will be visible between the odd and even slices. When odd and even slices are acquired closer in time (in ascending/descending mode, or interleaved but sequentially within the same b-vector), this artifact is not visible (right). Such an artifact could be problematic for image registration with regularization along the S-I axis, or for performing diffusion tractography. (b) $b=0$ image from a DWI scan acquired with poor shimming and resulting signal dropout. (c) Another example of poor shimming resulting in sub-efficient fat saturation, with the fat being aliased on top of the SC. Here we show the mean DWI scan of a subject. (d) Effect of pulsatile effects on a non-cardiac gated acquisition. Diffusion-weighted scans (sagittal view) acquired at three b-vecs fairly orthogonal to the SC (i.e., diffusion-specific signal attenuation should be minimum in the SC), showing abrupt signal drop at a few slices (red arrows), likely due to cardiac-related pulsatile effects. 


\section{Anticipated Results}

In this section we show images of the same subject acquired across the three vendors. Additional examples of good quality data with interactive $3 \mathrm{D}$ visualization are shown in the spine generic website ${ }^{133}$. The interactive embedding in the website is powered by Brainsprite ${ }^{134}$.

\section{Good quality T1w scans (step 6)}
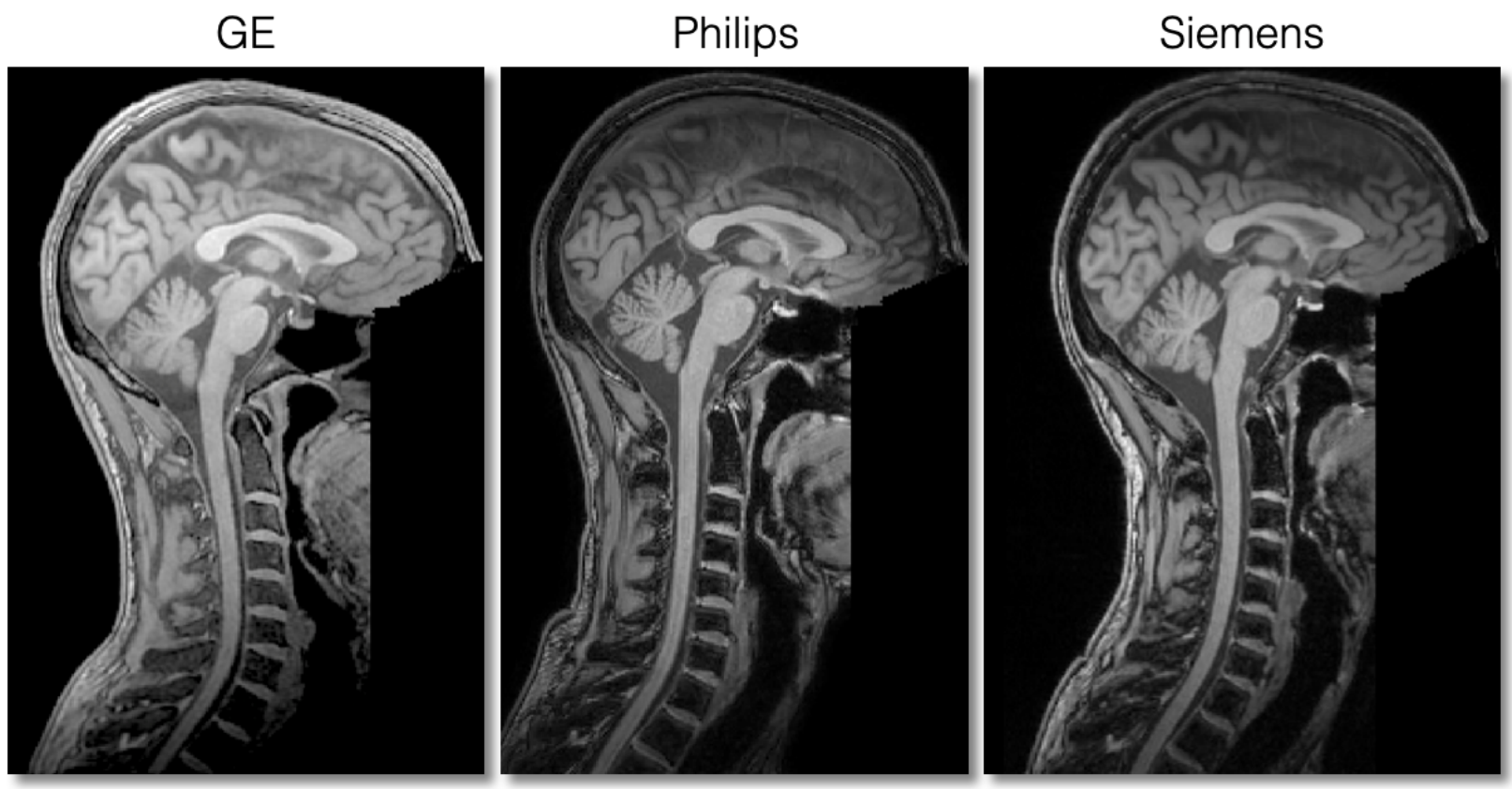

Figure 17. Sagittal views of good quality T1w scans for each vendor.

${ }^{133} \mathrm{https}$ ://spine-generic.readthedocs.io/en/latest/data-acquisition.html\#example-of-datasets

134 https://brainsprite.github.io/ 
Good quality T2w scans (step 7)
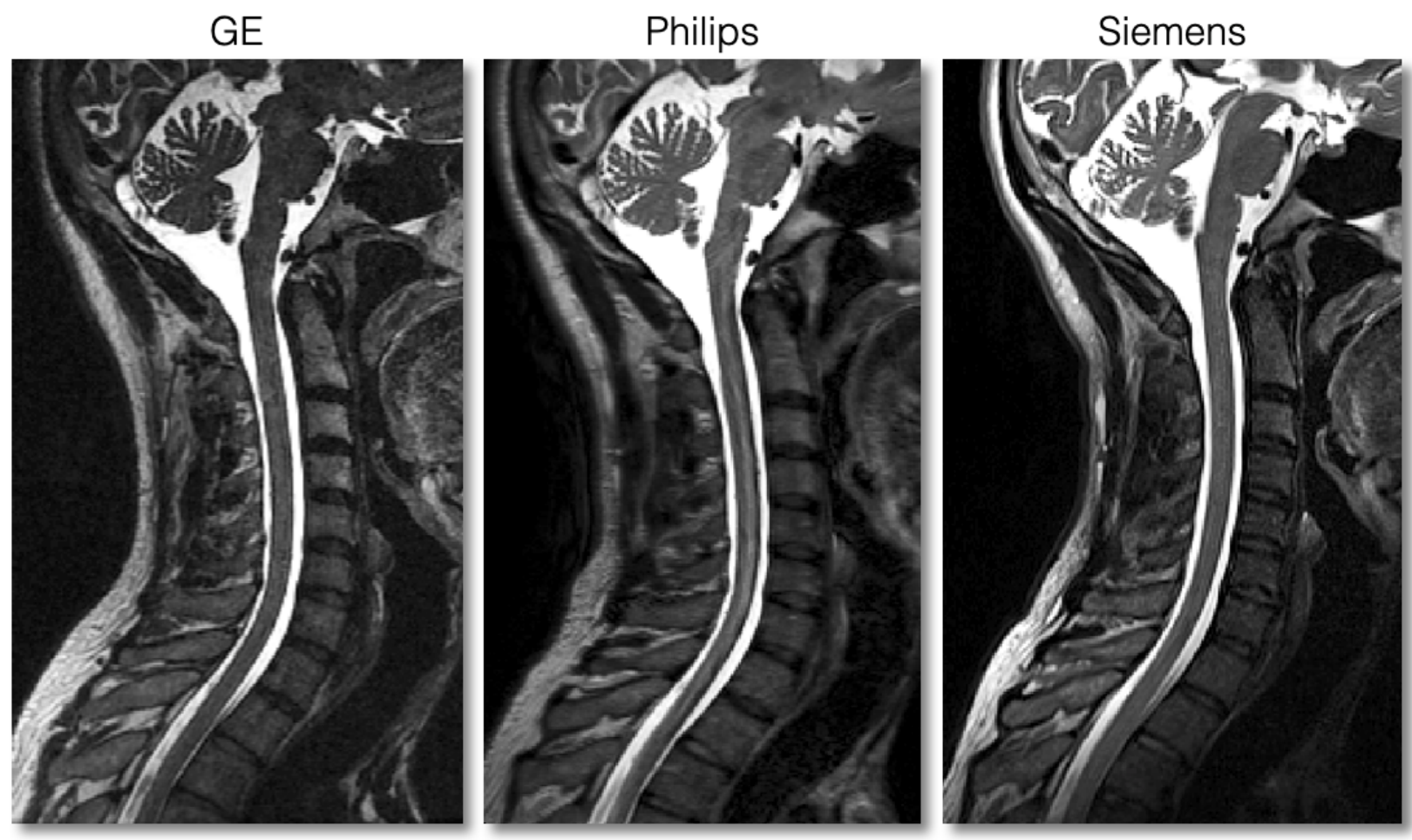

Figure 18. Sagittal views of good quality T2w scans for each vendor.

Good quality ME-GRE scans (step 8)
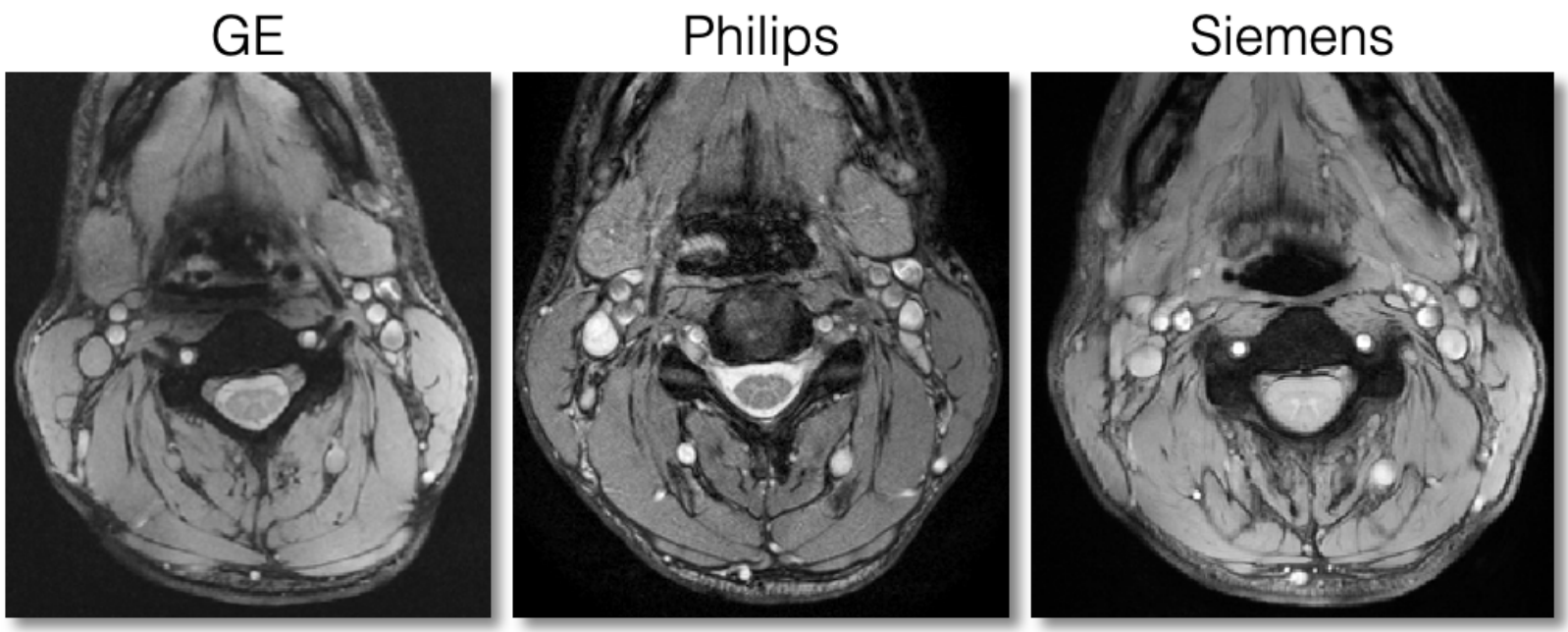

Figure 19. Sagittal views of good quality ME-GRE scans for each vendor. 


\section{Good quality MT scans (step 9)}
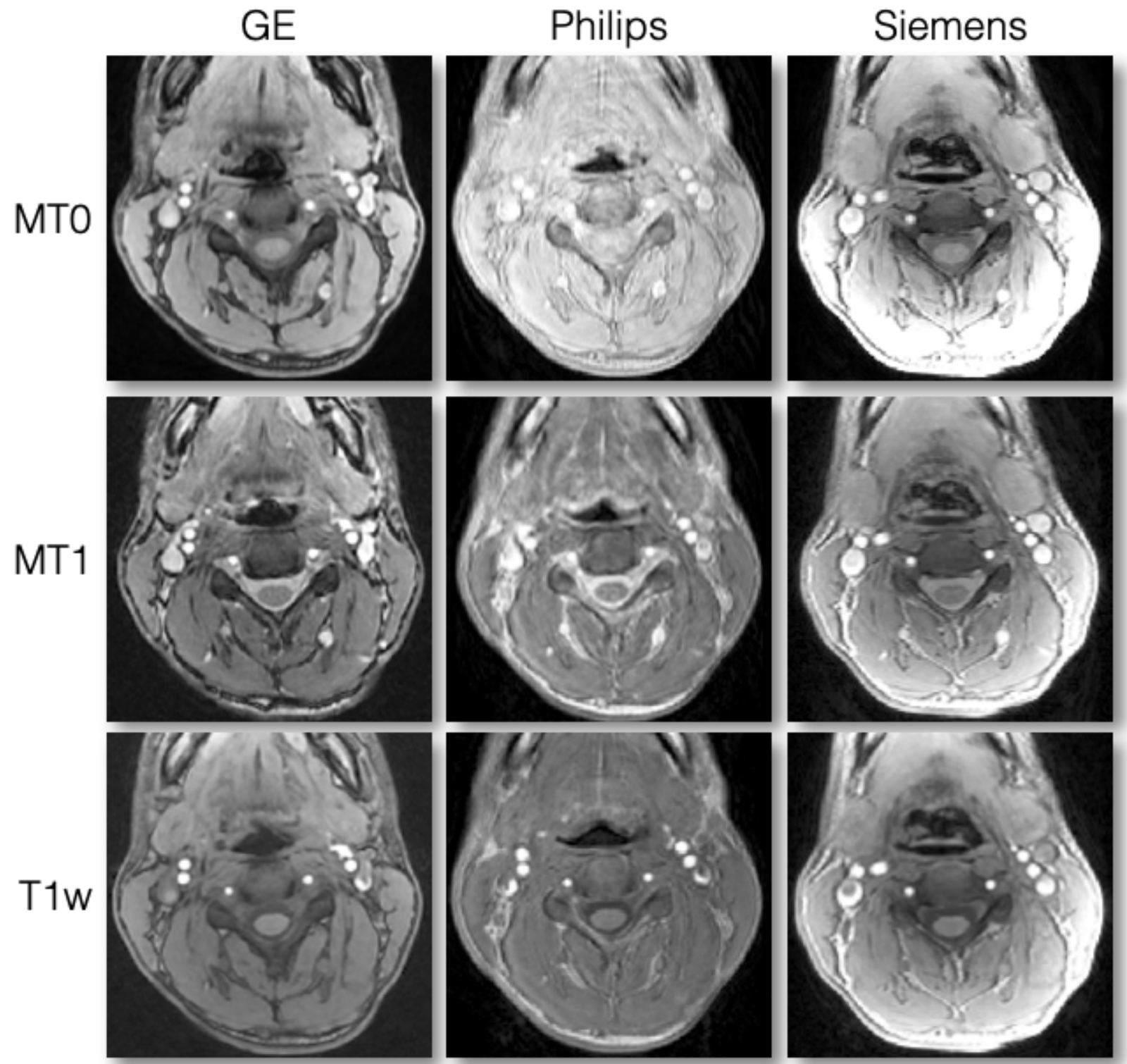

Figure 20. Axial views of good quality data for MTO, MT1 and T1w scans. Notice the slight motion artifact on the Philips MTO scan. Also notice the strong signal intensity at the periphery of the tissue on the Siemens scans, which is due to the inactivation of the intensity bias filter. This filter is not relevant when computing qMRI metrics such as MTR or MTsat. 


\section{Good quality DWI scans (step 10)}

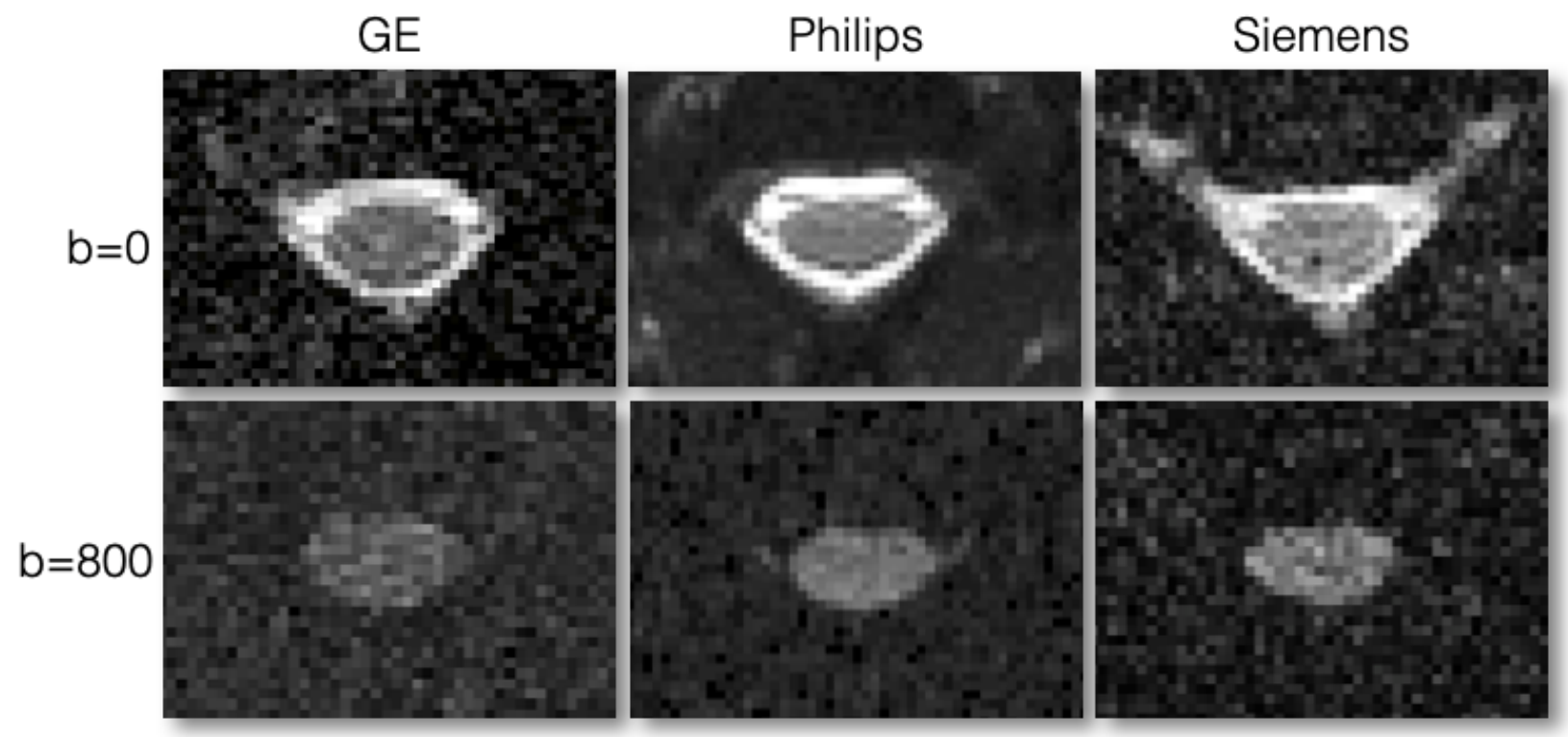

Figure 21. Axial views of good quality data for DWI scans at $b=0 \mathrm{~s} / \mathrm{mm}^{2}$ (top row) and $b=800$ $\mathrm{s} / \mathrm{mm}^{2}$ (bottom row). The DW image corresponds to a diffusion gradient vector fairly orthogonal to the cord axis, hence the visible spinal cord. When the diffusion gradient is oriented quasi-parallel to the cord, the signal in the cord almost vanishes. Notice the different noise patterns across the vendors, which is due to the different types of filters applied. These filters were present in the old version of the protocol and were removed in the latest version. 


\section{Acknowledgements}

We thank Gerald Moran and Bart Schraa (Siemens Healthcare), Suchandrima Banerjee and Naoyuki Takei (GE Healthcare) for sharing proprietary information and helping with setting up vendor-specific protocols, Carollyn Hurst, André Cyr, Arnaud Boré and Pierre Bellec (Functional Neuroimaging Unit), Charles Tremblay (Polytechnique Montreal), Antonys Melek and Habib Benali (PERFORM center, Concordia University), Ives Levesque (McGill University), Carol Nguyen (University of Minnesota), Prof. Shigeki Aoki (Juntendo University Hospital) for helping with data acquisitions, Compute Ontario (https://computeontario.ca/) and Compute Canada (www.computecanada.ca) for providing the supercomputer infrastructure and all the volunteers who participated in the Spinal Cord MRI Public Database.

Funded by the Canada Research Chair in Quantitative Magnetic Resonance Imaging [950-230815], the Canadian Institute of Health Research [CIHR FDN-143263], the Canada Foundation for Innovation [32454, 34824], the Fonds de Recherche du Québec - Santé [28826], the Fonds de Recherche du Québec - Nature et Technologies [2015-PR-182754], the Natural Sciences and Engineering Research Council of Canada [435897-2013], the Canada First Research Excellence Fund (IVADO and TransMedTech), the Quebec Biolmaging Network [5886], Spinal Research (UK), Wings for Life (Austria, \#169111) and Craig H. Neilsen Foundation (USA) for the INSPIRED project, the National Institutes of Health (NIH) through grants R00EB016689 and R01EB027779 (R.L.B.), the Instituto Investigación Carlos III (Spain, PI18/00823), the Czech Health Research Council grant n. NV18-04-00159, the Ministry of Health, Czech Republic - conceptual development of research organization (FNBr, 65269705), the National Imaging Facility and Queensland NMR Network (UQ), and SpinalCure Australia (M.J.R.), the European Research Council under the European Union's Seventh Framework Programme (FP7/2007-2013) / ERC grant agreement $n^{\circ}$ 616905; European Union's Horizon 2020 research and innovation programme under the grant agreement No 681094, and the Swiss State Secretariat for Education, Research and Innovation (SERI) under contract number 15.0137; BMBF (01EW1711A \& B) in the framework of ERA-NET NEURON, the European Union's Horizon 2020 research and innovation programme under grant agreement No. 634541, the Engineering and Physical Sciences Research Council (R006032/1, M020533/1) and Rosetrees Trust (UK), UK Multiple Sclerosis Society (892/08, 77/2017), NIHR Biomedical Research Centres, UCLH, the Italian Ministry of Health Young Researcher Grant 2013 (GR-2013-02358177), the FISR Project "Tecnopolo di nanotecnologia e fotonica per la medicina di precisione"(funded by MIUR/CNR, CUP B83B17000010001), TECNOMED project (funded by Regione Puglia, CUP B84I18000540002), Million Dollar Bike Ride from the University of Pennsylvania (MDBR-17-123-MPS), investigator-initiated PREdICT study at the Vall d'Hebron Institute of Oncology (Barcelona), funded by AstraZeneca and CRIS Cancer Foundation, the Wellcome Trust (UK) (203139/Z/16/Z), Systems, Technologies and Applications for Radiofrequency and Communications (STARaCOM) and Swiss National Science Foundation (PCEFP3_181362/1). The content is solely the responsibility of the authors and does not necessarily represent the official views of the NIH. 


\section{References}

1. Cercignani, M., Dowell, N. G. \& Tofts, P. S. Quantitative MRI of the Brain: Principles of Physical Measurement, Second edition. (CRC Press, 2018).

2. Cohen-Adad, J. \& Wheeler-Kingshott, C. Quantitative MRI of the Spinal Cord. (2014).

3. Wheeler-Kingshott, C. A. et al. The current state-of-the-art of spinal cord imaging: applications. Neuroimage 84, 1082-1093 (2014).

4. Stroman, P. W. et al. The current state-of-the-art of spinal cord imaging: methods. Neuroimage 84, 1070-1081 (2014).

5. Cohen-Adad, J. \& Wald, L. L. Array Coils. Quantitative MRI of the Spinal Cord 59-67 (2014) doi:10.1016/b978-0-12-396973-6.00005-8.

6. Barry, R. L., Vannesjo, S. J., By, S., Gore, J. C. \& Smith, S. A. Spinal cord MRI at 7T. Neuroimage 168, 437-451 (2018).

7. Saritas, E. U., Holdsworth, S. J. \& Bammer, R. Susceptibility artifacts. In: Quantitative MRI of the Spinal Cord, Elsevier, J. Cohen-Adad and CAM Wheeler-Kingshott, eds. 91-104 (2014).

8. Bonati, U. et al. Cervical cord and brain grey matter atrophy independently associate with long-term MS disability. J. Neurol. Neurosurg. Psychiatry 82, 471-472 (2011).

9. Cohen, A. B. et al. The relationships among MRI-defined spinal cord involvement, brain involvement, and disability in multiple sclerosis. J. Neuroimaging 22, 122-128 (2012).

10. Kearney, H. et al. Magnetic resonance imaging correlates of physical disability in relapse onset multiple sclerosis of long disease duration. Mult. Scler. 20, 72-80 (2014).

11. Lukas, C. et al. Relevance of spinal cord abnormalities to clinical disability in multiple sclerosis: MR imaging findings in a large cohort of patients. Radiology 269, 542-552 (2013).

12. Branco, L. M. T. et al. Spinal cord atrophy correlates with disease duration and severity in amyotrophic lateral sclerosis. Amyotroph. Lateral Scler. Frontotemporal Degener. 15, 93-97 (2014).

13. El Mendili, M.-M. et al. Multi-parametric spinal cord MRI as potential progression marker in amyotrophic lateral sclerosis. PLoS One 9, e95516 (2014).

14. de Albuquerque, M. et al. Longitudinal evaluation of cerebral and spinal cord damage in Amyotrophic Lateral Sclerosis. Neuroimage Clin 14, 269-276 (2017). 
15. Querin, G. et al. Spinal cord multi-parametric magnetic resonance imaging for survival prediction in amyotrophic lateral sclerosis. Eur. J. Neurol. 24, 1040-1046 (2017).

16. Paquin, M.-Ê. et al. Spinal Cord Gray Matter Atrophy in Amyotrophic Lateral Sclerosis. AJNR Am. J. Neuroradiol. 39, 184-192 (2018).

17. van de Stadt, S. I. W. et al. Spinal cord atrophy as a measure of severity of myelopathy in adrenoleukodystrophy. J. Inherit. Metab. Dis. (2020) doi:10.1002/jimd.12226.

18. Kadanka, Z., Jr et al. Predictors of symptomatic myelopathy in degenerative cervical spinal cord compression. Brain Behav. 7, e00797 (2017).

19. Seif, M. et al. Cervical Cord Neurodegeneration in Traumatic and Non-Traumatic Spinal Cord Injury. J. Neurotrauma 37, 860-867 (2020).

20. De Leener, B. et al. SCT: Spinal Cord Toolbox, an open-source software for processing spinal cord MRI data. Neuroimage 145, 24-43 (2017).

21. Rasoanandrianina, $\mathrm{H}$. et al. Region-specific impairment of the cervical spinal cord (SC) in amyotrophic lateral sclerosis: A preliminary study using SC templates and quantitative MRI (diffusion tensor imaging/inhomogeneous magnetization transfer). NMR Biomed. 30, e3801 (2017).

22. Schmierer, K., Scaravilli, F., Altmann, D. R., Barker, G. J. \& Miller, D. H. Magnetization transfer ratio and myelin in postmortem multiple sclerosis brain. Ann. Neurol. 56, 407-415 (2004).

23. Fatemi, A. et al. Magnetization transfer MRI demonstrates spinal cord abnormalities in adrenomyeloneuropathy. Neurology 64, 1739-1745 (2005).

24. Lema, A. et al. A Comparison of Magnetization Transfer Methods to Assess Brain and Cervical Cord Microstructure in Multiple Sclerosis. J. Neuroimaging 27, 221-226 (2017).

25. Cohen-Adad, J. et al. Demyelination and degeneration in the injured human spinal cord detected with diffusion and magnetization transfer MRI. Neuroimage 55, 1024-1033 (2011).

26. Martin, A. R. et al. Translating state-of-the-art spinal cord MRI techniques to clinical use: A systematic review of clinical studies utilizing DTI, MT, MWF, MRS, and fMRI. Neuroimage Clin 10, 192-238 (2016).

27. David, G. et al. Traumatic and nontraumatic spinal cord injury: pathological insights from neuroimaging. Nature Reviews Neurology vol. 15 718-731 (2019).

28. Cadotte, D. W., Akbar, M. A., Fehlings, M. G., Stroman, P. W. \& Cohen-Adad, J. What Has Been Learned from Magnetic Resonance Imaging Examination of the Injured Human Spinal Cord: A Canadian Perspective. J. Neurotrauma 35, 1942-1957 (2018). 
29. Huffnagel, I. C. et al. Longitudinal diffusion MRI as surrogate outcome measure for myelopathy in adrenoleukodystrophy. Neurology 93, e2133-e2143 (2019).

30. Martin, A. R. et al. Can microstructural MRI detect subclinical tissue injury in subjects with asymptomatic cervical spinal cord compression? A prospective cohort study. BMJ Open 8 , e019809 (2018).

31. Labounek, R. et al. HARDI-ZOOMit protocol improves specificity to microstructural changes in presymptomatic myelopathy. Sci. Rep. 10, 17529 (2020).

32. Levy, S. et al. White matter atlas of the human spinal cord with estimation of partial volume effect. Neuroimage 119, 262-271 (2015).

33. Feaster, D. J., Mikulich-Gilbertson, S. \& Brincks, A. M. Modeling site effects in the design and analysis of multi-site trials. Am. J. Drug Alcohol Abuse 37, 383-391 (2011).

34. Fratini, M. et al. Multiscale Imaging Approach for Studying the Central Nervous System: Methodology and Perspective. Front. Neurosci. 14, 72 (2020).

35. Grussu, F. et al. Multi-parametric quantitative in vivo spinal cord MRI with unified signal readout and image denoising. Neuroimage 217, 116884 (2020).

36. Gros, C. et al. Automatic segmentation of the spinal cord and intramedullary multiple sclerosis lesions with convolutional neural networks. Neuroimage 184, 901-915 (2019).

37. Papinutto, N. \& Henry, R. G. Evaluation of intra-and interscanner reliability of MRI protocols for spinal cord gray matter and total cross-sectional area measurements. J. Magn. Reson. Imaging 49, 1078-1090 (2019).

38. Perone, C. S., Ballester, P., Barros, R. C. \& Cohen-Adad, J. Unsupervised domain adaptation for medical imaging segmentation with self-ensembling. Neuroimage 194, 1-11 (2019).

39. Perone, C. S., Calabrese, E. \& Cohen-Adad, J. Spinal cord gray matter segmentation using deep dilated convolutions. Sci. Rep. 8, 5966 (2018).

40. Lévy, S. et al. Test-retest reliability of myelin imaging in the human spinal cord: Measurement errors versus region- and aging-induced variations. PLOS ONE vol. 13 e0189944 (2018).

41. Gros, C. et al. Automatic spinal cord localization, robust to MRI contrasts using global curve optimization. Med. Image Anal. (2017).

42. Duval, T., Smith, V., Stikov, N., Klawiter, E. C. \& Cohen-Adad, J. Scan-rescan of axcaliber, macromolecular tissue volume, and g-ratio in the spinal cord. Magnetic Resonance in Medicine vol. 79 2759-2765 (2018). 
43. De Leener, B. et al. PAM50: Unbiased multimodal template of the brainstem and spinal cord aligned with the ICBM152 space. Neuroimage 165, 170-179 (2018).

44. Prados, F. et al. Spinal cord grey matter segmentation challenge. Neuroimage 152, 312-329 (2017).

45. Leener, B. D. et al. Topologically preserving straightening of spinal cord MRI. Journal of Magnetic Resonance Imaging vol. 46 1209-1219 (2017).

46. Duval, T. et al. g-Ratio weighted imaging of the human spinal cord in vivo. Neuroimage 145, Part A, 11-23 (2017).

47. Dupont, S. M. et al. Fully-integrated framework for the segmentation and registration of the spinal cord white and gray matter. Neuroimage (2017)

doi:10.1016/j.neuroimage.2016.09.026.

48. Daniel Papp, Alex Kenneth Smith, Romina Mariano, Stuart Clare. High-resolution quantitative maps of magnetisation transfer, R1 and R2* of the cervical spinal cord in clinically feasible acquisition time using vendor-provided sequences. in Proceedings of the 27th Annual Meeting of ISMRM, Montreal, Canada.

49. Vahdat, S. et al. Resting-state brain and spinal cord networks in humans are functionally integrated. PLoS Biol. 18, e3000789 (2020).

50. DiNuzzo, M. et al. Towards a Standard Pipeline for the Analysis of Human Spinal Cord fMRI Data Series. in Proceedings of the 27th Annual Meeting of ISMRM, Montreal, Canada (2019).

51. Moccia, M. et al. Longitudinal spinal cord atrophy in multiple sclerosis using the generalized boundary shift integral. Ann. Neurol. 86, 704-713 (2019).

52. Prados, F. et al. Generalised boundary shift integral for longitudinal assessment of spinal cord atrophy. Neurolmage vol. 209116489 (2020).

53. J. Oh, N. Arbour, F. Giuliani, S. Kolind, L. Lynd, R.-A. Marrie, L. Metz, S. Patten, A. Prat, A. Schabas, P. Smyth, R. Tam, A. Traboulsee, W. Yong. The Canadian prospective cohort (canproco) study to understand progression in multiple sclerosis: rationale, aims, and study design. in ECTRIMS 2019.

54. Nestrasil, I. et al. Cervical spinal cord diffusion MRI and intraspinal space restriction at the occipito-cervical junction in mucopolysacharidoses patients. in Proceedings of the 27th Annual Meeting of ISMRM, Montreal, Canada (2019).

55. Querin, G. et al. The spinal and cerebral profile of adult spinal-muscular atrophy: A multimodal imaging study. Neuroimage Clin 21, 101618 (2019).

56. Querin, G. et al. Presymptomatic spinal cord pathology in c9orf72 mutation carriers: A 
longitudinal neuroimaging study. Ann. Neurol. 86, 158-167 (2019).

57. Martin, A. R. et al. Monitoring for myelopathic progression with multiparametric quantitative MRI. PLoS One 13, e0195733 (2018).

58. Martin, A. R. et al. A Novel MRI Biomarker of Spinal Cord White Matter Injury: T2*-Weighted White Matter to Gray Matter Signal Intensity Ratio. AJNR Am. J. Neuroradiol. 38, 1266-1273 (2017).

59. Martin, A. R. et al. Clinically Feasible Microstructural MRI to Quantify Cervical Spinal Cord Tissue Injury Using DTI, MT, and T2*-Weighted Imaging: Assessment of Normative Data and Reliability. AJNR Am. J. Neuroradiol. 38, 1257-1265 (2017).

60. Karbasforoushan, H., Cohen-Adad, J. \& Dewald, J. P. A. Brainstem and spinal cord MRI identifies altered sensorimotor pathways post-stroke. Nat. Commun. 10, 3524 (2019).

61. Seif, M., Gandini Wheeler-Kingshott, C. A., Cohen-Adad, J., Flanders, A. E. \& Freund, P. Guidelines for the conduct of clinical trials in spinal cord injury: Neuroimaging biomarkers. Spinal Cord 57, 717-728 (2019).

62. Bagnato, F. et al. Imaging Mechanisms of Disease Progression in Multiple Sclerosis: Beyond Brain Atrophy. J. Neuroimaging 30, 251-266 (2020).

63. Tinnermann, A., Büchel, C. \& Cohen-Adad, J. Cortico-spinal imaging to study pain. Neuroimage 224, 117439 (2020).

64. Cohen-Adad, J. Microstructural imaging in the spinal cord and validation strategies. Neuroimage In press, (2018).

65. Topfer, R. et al. A 24-channel shim array for the human spinal cord: Design, evaluation, and application. Magn. Reson. Med. 76, 1604-1611 (2016).

66. Topfer, R., Foias, A., Stikov, N. \& Cohen-Adad, J. Real-time correction of respiration-induced distortions in the human spinal cord using a 24-channel shim array. Magn. Reson. Med. 80, 935-946 (2018).

67. Islam, H., Law, C. S. W., Weber, K. A., Mackey, S. C. \& Glover, G. H. Dynamic per slice shimming for simultaneous brain and spinal cord fMRI. Magnetic Resonance in Medicine vol. $81825-838$ (2019).

68. Finsterbusch, J., Eippert, F. \& Buchel, C. Single, slice-specific z-shim gradient pulses improve T2*-weighted imaging of the spinal cord. Neuroimage 59, 2307-2315 (2012).

69. Finsterbusch, J. B0 Inhomogeneity and Shimming. In: Quantitative MRI of the Spinal Cord, Elsevier, J. Cohen-Adad and CAM Wheeler-Kingshott, eds. 68-88 (2014).

70. Verma, T. \& Cohen-Adad, J. Effect of respiration on the B0 field in the human spinal cord at 
3T. Magn. Reson. Med. 72, 1629-1636 (2014).

71. Vannesjo, S. J., Miller, K. L., Clare, S. \& Tracey, I. Spatiotemporal characterization of breathing-induced BOfield fluctuations in the cervical spinal cord at 7T. Neuroimage 167, 191-202 (2018).

72. van Gelderen, P., de Zwart, J. A., Starewicz, P., Hinks, R. S. \& Duyn, J. H. Real-time shimming to compensate for respiration-induced B0 fluctuations. Magn. Reson. Med. 57, 362-368 (2007).

73. Porter, D. A. \& Heidemann, R. M. High resolution diffusion-weighted imaging using readout-segmented echo-planar imaging, parallel imaging and a two-dimensional navigator-based reacquisition. Magn. Reson. Med. 62, 468-475 (2009).

74. Barry, R. L., Klassen, L. M., Williams, J. M. \& Menon, R. S. Hybrid two-dimensional navigator correction: a new technique to suppress respiratory-induced physiological noise in multi-shot echo-planar functional MRI. Neuroimage 39, 1142-1150 (2008).

75. Rasoanandrianina, $\mathrm{H}$. et al. Regional T 1 mapping of the whole cervical spinal cord using an optimized MP2RAGE sequence. NMR in Biomedicine (2019) doi:10.1002/nbm.4142.

76. Boudreau, M. et al. B1 mapping for bias-correction in quantitative T1 imaging of the brain at 3T using standard pulse sequences. J. Magn. Reson. Imaging 46, 1673-1682 (2017).

77. Helms, G. Correction for residual effects of B1+ inhomogeniety on MT saturation in FLASH-based multi-parameter mapping of the brain. in.

78. Gilbert, K. M., Curtis, A. T., Gati, J. S., Klassen, L. M. \& Menon, R. S. A radiofrequency coil to facilitate $\mathrm{B}_{1}{ }^{+}$shimming and parallel imaging acceleration in three dimensions at $7 \mathrm{~T}$. NMR Biomed. 24, 815-823 (2011).

79. Chung, S., Kim, D., Breton, E. \& Axel, L. Rapid B1+ mapping using a preconditioning RF pulse with TurboFLASH readout. Magn. Reson. Med. 64, 439-446 (2010).

80. Lutti, A., Hutton, C., Finsterbusch, J., Helms, G. \& Weiskopf, N. Optimization and validation of methods for mapping of the radiofrequency transmit field at 3T. Magn. Reson. Med. 64, 229-238 (2010).

81. Yarnykh, V. L. Actual flip-angle imaging in the pulsed steady state: a method for rapid three-dimensional mapping of the transmitted radiofrequency field. Magn. Reson. Med. 57, 192-200 (2007).

82. Nehrke, K. \& Börnert, P. DREAM—a novel approach for robust, ultrafast, multislice B1 mapping. Magn. Reson. Med. 68, 1517-1526 (2012).

83. Sacolick, L. I., Wiesinger, F., Hancu, I. \& Vogel, M. W. B1 mapping by Bloch-Siegert shift. 
Magn. Reson. Med. 63, 1315-1322 (2010).

84. Nelson, F. et al. Improved identification of intracortical lesions in multiple sclerosis with phase-sensitive inversion recovery in combination with fast double inversion recovery MR imaging. AJNR Am. J. Neuroradiol. 28, 1645-1649 (2007).

85. Poonawalla, A. H., Hou, P., Nelson, F. A., Wolinsky, J. S. \& Narayana, P. A. Cervical spinal cord lesions in multiple sclerosis: T1-weighted inversion-recovery MR imaging with phase-sensitive reconstruction. Radiology 246, 258-264 (2008).

86. Alcaide-Leon, P. et al. Quantitative spinal cord MRI in radiologically isolated syndrome. Neurology - Neuroimmunology Neuroinflammation vol. 5 e436 (2018).

87. Kearney, H., Miszkiel, K. A., Yiannakas, M. C., Ciccarelli, O. \& Miller, D. H. A pilot MRI study of white and grey matter involvement by multiple sclerosis spinal cord lesions. Mult. Scler. Relat. Disord. 2, 103-108 (2013).

88. Kearney, H. et al. Improved MRI quantification of spinal cord atrophy in multiple sclerosis. J. Magn. Reson. Imaging 39, 617-623 (2014).

89. Papinutto, N. et al. 2D phase-sensitive inversion recovery imaging to measure in vivo spinal cord gray and white matter areas in clinically feasible acquisition times. J. Magn. Reson. Imaging 42, 698-708 (2015).

90. Schlaeger, R. et al. Spinal cord gray matter atrophy correlates with multiple sclerosis disability. Ann. Neurol. 76, 568-580 (2014).

91. Bonacchi, R. et al. Clinical Relevance of Multiparametric MRI Assessment of Cervical Cord Damage in Multiple Sclerosis. Radiology 200430 (2020) doi:10.1148/radiol.2020200430.

92. Olney, N. T. et al. Measurement of spinal cord atrophy using phase sensitive inversion recovery (PSIR) imaging in motor neuron disease. PLoS One 13, e0208255 (2018).

93. Papinutto, N. et al. Age, Gender and Normalization Covariates for Spinal Cord Gray Matter and Total Cross-Sectional Areas at Cervical and Thoracic Levels: A 2D Phase Sensitive Inversion Recovery Imaging Study. PLoS One 10, e0118576 (2015).

94. Papinutto, N. et al. Intersubject Variability and Normalization Strategies for Spinal Cord Total Cross-Sectional and Gray Matter Areas. J. Neuroimaging 30, 110-118 (2020).

95. Fradet, L., Arnoux, P.-J., Ranjeva, J.-P., Petit, Y. \& Callot, V. Morphometrics of the entire human spinal cord and spinal canal measured from in vivo high-resolution anatomical magnetic resonance imaging. Spine 39, E262-9 (2014).

96. Yiannakas, M. C., Kakar, P., Hoy, L. R., Miller, D. H. \& Wheeler-Kingshott, C. A. M. The use of the lumbosacral enlargement as an intrinsic imaging biomarker: feasibility of grey matter and white matter cross-sectional area measurements using MRI at 3T. PLoS One 9, 
e105544 (2014).

97. Tillieux, P. D. et al. A pneumatic phantom for mimicking respiration-induced artifacts in spinal MRI. Magnetic Resonance in Medicine vol. 79 600-605 (2018).

98. Massire, A. et al. Feasibility of single-shot multi-level multi-angle diffusion tensor imaging of the human cervical spinal cord at 7T. Magn. Reson. Med. 80, 947-957 (2018).

99. Massire, A. et al. High-resolution multi-parametric quantitative magnetic resonance imaging of the human cervical spinal cord at 7T. Neuroimage 143, 58-69 (2016).

100.Li, D. K. B. et al. Developing a Universally Useful, Useable and Used Standardized MRI Protocol for Patients with Multiple Sclerosis. in Proceedings of the 28th Annual Meeting of ISMRM, Sydney, Australia.

101. Wilkinson, M. D. et al. The FAIR Guiding Principles for scientific data management and stewardship. Sci Data 3, 160018 (2016).

102.Stikov, N., Trzasko, J. D. \& Bernstein, M. A. Reproducibility and the future of MRI research. Magn. Reson. Med. 82, 1981-1983 (2019).

103.Xu, J. et al. Improved in vivo diffusion tensor imaging of human cervical spinal cord. Neuroimage 67, 64-76 (2013).

104.Summers, P. E., Brooks, J. \& Cohen-Adad, J. Spinal Cord fMRI. in Quantitative MRI of the Spinal Cord (eds. Cohen-Adad, J. \& M, W.-K. C. A.) 221-236 (Elsevier, 2014).

105. Glasser, M. F. et al. The minimal preprocessing pipelines for the Human Connectome Project. Neuroimage 80, 105-124 (2013).

106. Helms, G., Dathe, H., Kallenberg, K. \& Dechent, P. High-resolution maps of magnetization transfer with inherent correction for RF inhomogeneity and $\mathrm{T} 1$ relaxation obtained from $3 \mathrm{D}$ FLASH MRI. Magn. Reson. Med. 60, 1396-1407 (2008).

107.5th Spinal Cord MRI Workshop. http://www.spinalcordmri.org/2018/06/22/workshop.html.

108.Song, S. K. et al. Demyelination increases radial diffusivity in corpus callosum of mouse brain. Neuroimage 26, 132-140 (2005).

109.Jones, D. K. \& Basser, P. J. 'Squashing peanuts and smashing pumpkins': how noise distorts diffusion-weighted MR data. Magn. Reson. Med. 52, 979-993 (2004).

110. Saritas, E. U., Cunningham, C. H., Lee, J. H., Han, E. T. \& Nishimura, D. G. DWI of the spinal cord with reduced FOV single-shot EPI. Magn. Reson. Med. 60, 468-473 (2008).

111. Finsterbusch, J. High-resolution diffusion tensor imaging with inner field-of-view EPI. J. Magn. Reson. Imaging 29, 987-993 (2009). 
112. Wilm, B. J. et al. Diffusion-weighted imaging of the entire spinal cord. NMR Biomed. 22, 174-181 (2009).

113. Jeong, E.-K., Kim, S.-E., Guo, J., Kholmovski, E. G. \& Parker, D. L. High-resolution DTI with 2D interleaved multislice reduced FOV single-shot diffusion-weighted EPI (2D ss-rFOV-DWEPI). Magnetic Resonance in Medicine vol. 54 1575-1579 (2005).

114. Samson, R. S. et al. ZOOM or Non-ZOOM? Assessing Spinal Cord Diffusion Tensor Imaging Protocols for Multi-Centre Studies. PLoS One 11, e0155557 (2016).

115. Summers P. Staempfli T. Jaermann S. Kwiecinski S. Kollias. A Preliminary Study of the Effects of Trigger Timing on Diffusion Tensor Imaging of the Human. AJNR 27, (2006).

116. Pfeuffer, J. et al. Zoomed functional imaging in the human brain at 7 Tesla with simultaneous high spatial and high temporal resolution. Neuroimage 17, 272-286 (2002).

117. Kim, J., Yang, C. \& Xu, J. Automated spinal cord diffusion MRI quality assurance using deep neural network. in.

118. Yiannakas, M. C. et al. Feasibility of grey matter and white matter segmentation of the upper cervical cord in vivo: A pilot study with application to magnetisation transfer measurements. Neuroimage 63, 1054-1059 (2012).

119. Bellec, P. \& Boyle, J. A. Bridging the gap between perception and action: the case for neuroimaging, Al and video games. (2019) doi:10.31234/osf.io/3epws. 